\title{
Quasilinear and Hessian type equations with exponential reaction and measure data*
}

\author{
Nguyen Quoc Hung ${ }^{\dagger}$ \\ Laurent Véron \\ Laboratoire de Mathématiques et Physique Théorique, \\ Université François Rabelais, Tours, FRANCE
}

\section{Contents}

1 Introduction $\quad 2$

2 Estimates on potentials and Wolff integral equations 9

3 Quasilinear Dirichlet problems

4 p-superharmonic functions and quasilinear equations in $\mathbb{R}^{N} \quad 23$

5 Hessian equations $\quad 25$

\begin{abstract}
We prove existence results concerning equations of the type $-\Delta_{p} u=P(u)+\mu$ for $p>1$ and $F_{k}[-u]=P(u)+\mu$ with $1 \leq k<\frac{N}{2}$ in a bounded domain $\Omega$ or the whole $\mathbb{R}^{N}$, where $\mu$ is a positive Radon measure and $P(u) \sim e^{a u^{\beta}}$ with $a>0$ and $\beta \geq 1$. Sufficient conditions for existence are expressed in terms of the fractional maximal potential of $\mu$. Two-sided estimates on the solutions are obtained in terms of some precise Wolff potentials of $\mu$. Necessary conditions are obtained in terms of Orlicz capacities. We also establish existence results for a general Wolff potential equation under the form $u=\mathbf{W}_{\alpha, p}^{R}[P(u)]+f$ in $\mathbb{R}^{N}$, where $0<R \leq \infty$ and $f$ is a positive integrable function.
\end{abstract}

2010 Mathematics Subject Classification. 31C15, 32 F10, 35J92, 35R06, 46E30.

${ }^{*}$ To appear Arch. Rat. Mech. Anal.

${ }^{\dagger}$ E-mail address: Hung.Nguyen-Quoc@lmpt.univ-tours.fr

${ }^{\ddagger}$ E-mail address: Laurent.Veron@lmpt.univ-tours.fr, Corresponding author. 
Key words: quasilinear elliptic equations, Hessian equations, Wolff potential, maximal functions, Borel measures, Orlicz capacities.

\section{Introduction}

Let $\Omega \subset \mathbb{R}^{N}$ be either a bounded domain or the whole $\mathbb{R}^{N}, p>1$ and $k \in\{1,2, \ldots, N\}$. We denote by

$$
\Delta_{p} u:=\operatorname{div}\left(|\nabla u|^{p-2} \nabla u\right)
$$

the p-Laplace operator and by

$$
F_{k}[u]=\sum_{1 \leq j_{1}<j_{2}<\ldots<j_{k} \leq N} \lambda_{j_{1}} \lambda_{j_{2}} \ldots \lambda_{j_{k}}
$$

the k-Hessian operator where $\lambda_{1}, \ldots, \lambda_{N}$ are the eigenvalues of the Hessian matrix $D^{2} u$. Let $\mu$ be a positive Radon measure in $\Omega$; our aim is to study the existence of nonnegative solutions to the following boundary value problems if $\Omega$ is bounded,

$$
\begin{aligned}
-\Delta_{p} u & =P(u)+\mu & & \text { in } \Omega, \\
u & =0 & & \text { on } \partial \Omega,
\end{aligned}
$$

and

$$
\begin{aligned}
F_{k}[-u] & =P(u)+\mu & & \text { in } \Omega, \\
u & =\varphi & & \text { on } \partial \Omega,
\end{aligned}
$$

where $P$ is an exponential function. If $\Omega=\mathbb{R}^{N}$, we consider the same equations, but the boundary conditions are replaced by $\inf _{\mathbb{R}^{N}} u=0$. When $P(r)=r^{q}$ with $q>p-1$, Phuc and Verbitsky published a seminal article [20] on the solvability of the corresponding problem (1.1). They obtained necessary and sufficient conditions involving Bessel capacities or Wolff potentials. For example, assuming that $\Omega$ is bounded, they proved that if $\mu$ has compact support in $\Omega$ it is equivalent to solve (1.1) with $P(r)=r^{q}$, or to have

$$
\mu(E) \leq c \operatorname{Cap}_{\mathbf{G}_{p}, \frac{q}{q+1-p}}(E) \quad \text { for all compact set } E \subset \Omega,
$$

where $c$ is a suitable positive constant and $\operatorname{Cap}_{\mathbf{G}_{p}, \frac{q}{q+1-p}}$ a Bessel capacity, or to have

$$
\int_{B}\left(\mathbf{W}_{1, p}^{2 R}\left[\mu_{B}\right](x)\right)^{q} d x \leq C \mu(B) \quad \text { for all ball } B \text { s.t. } B \cap \operatorname{supp} \mu \neq \emptyset
$$

where $R=\operatorname{diam}(\Omega)$. Other conditions are expressed in terms of Riesz potentials and maximal fractional potentials. Their construction is based upon sharp estimates of solutions of the nonhomogeneous problem

$$
\begin{aligned}
& -\Delta_{p} u=\omega \quad \text { in } \Omega, \\
& u=0 \quad \text { on } \partial \Omega \text {, }
\end{aligned}
$$

for positive measures $\omega$. We refer to [4, 5, 6, 7, 9, 13, 23, for the previous studies of these and other related results. Concerning the k-Hessian operator in a bounded $(k-1)$-convex domain $\Omega$, 
they proved that if $\mu$ has compact support and $\|\varphi\|_{L^{\infty}(\partial \Omega)}$ is small enough, the corresponding problem (1.2) with $P(r)=r^{q}$ with $q>k$ admits a nonnegative solution if and only if

$$
\mu(E) \leq c \operatorname{Cap}_{\mathbf{G}_{2 k}, \frac{q}{q-k}}(E) \quad \text { for all compact set } E \subset \Omega,
$$

or equivalently

$$
\int_{B}\left[\mathbf{W}_{\frac{2 k}{k+1}, k+1}^{2 R}\left[\mu_{B}(x)\right]\right]^{q} d x \leq C \mu(B) \quad \text { for all ball } B \text { s.t. } B \cap \operatorname{supp} \mu \neq \emptyset .
$$

The results concerning the linear case $p=2$ and $k=1$, can be found in 2, 3, 28. The main tools in their proofs are derived from recent advances in potential theory for nonlinear elliptic equations obtained by Kilpelainen and Malý [15, 16, Trudinger and Wang [24, 25, 26], and Labutin [18] thanks to whom the authors first provide global pointwise estimates for solutions of the homogeneous Dirichlet problems in terms of Wolff potentials of suitable order.

For $s>1,0 \leq \alpha<\frac{N}{s}, \eta \geq 0$ and $0<T \leq \infty$, we recall that the $T$-truncated Wolff potential of a positive Radon measure $\mu$ is defined in $\mathbb{R}^{N}$ by

$$
\mathbf{W}_{\alpha, s}^{T}[\mu](x)=\int_{0}^{T}\left(\frac{\mu\left(B_{t}(x)\right)}{t^{N-\alpha s}}\right)^{\frac{1}{s-1}} \frac{d t}{t},
$$

the T-truncated Riesz potential of a positive Radon measure $\mu$ by

$$
\mathbf{I}_{\alpha}^{T}[\mu](x)=\int_{0}^{T} \frac{\mu\left(B_{t}(x)\right)}{t^{N-\alpha}} \frac{d t}{t},
$$

and the $T$-truncated $\eta$-fractional maximal potential of $\mu$ by

$$
\mathbf{M}_{\alpha, T}^{\eta}[\mu](x)=\sup \left\{\frac{\mu\left(B_{t}(x)\right)}{t^{N-\alpha} h_{\eta}(t)}: 0<t \leq T\right\},
$$

where $h_{\eta}(t)=(-\ln t)^{-\eta} \chi_{\left(0,2^{-1}\right]}(t)+(\ln 2)^{-\eta} \chi_{\left[2^{-1}, \infty\right)}(t)$. If $\eta=0$, then $h_{\eta}=1$ and we denote by $\mathbf{M}_{\alpha, T}[\mu]$ the corresponding $T$-truncated fractional maximal potential of $\mu$. We also denote by $\mathbf{W}_{\alpha, s}[\mu]$ (resp $\mathbf{I}_{\alpha}[\mu], \mathbf{M}_{\alpha}^{\eta}[\mu]$ ) the $\infty$-truncated Wolff potential (resp Riesz Potential, $\eta-$ fractional maximal potential) of $\mu$. When the measures are only defined in an open subset $\Omega \subset \mathbb{R}^{N}$, they are naturally extended by 0 in $\Omega^{c}$. For $l \in \mathbb{N}^{*}$, we define the $l$-truncated exponential function

$$
H_{l}(r)=e^{r}-\sum_{j=0}^{l-1} \frac{r^{j}}{j !},
$$

and for $a>0$ and $\beta \geq 1$, we set

$$
P_{l, a, \beta}(r)=H_{l}\left(a r^{\beta}\right)
$$

We put

$$
Q_{p}(s)= \begin{cases}\sum_{q=l}^{\infty} \frac{s^{\frac{\beta q}{p-1}}}{q^{\frac{\beta q}{p-1}} q !} & \text { if } p \neq 2, \\ H_{l}\left(s^{\beta}\right) & \text { if } p=2,\end{cases}
$$


$Q_{p}^{*}(r)=\max \left\{r s-Q_{p}(s): s \geq 0\right\}$ is the complementary function to $Q_{p}$, and define the corresponding Bessel and Riesz capacities respectively by

$$
\operatorname{Cap}_{\mathbf{G}_{\alpha p}, Q_{p}^{*}}(E)=\inf \left\{\int_{\mathbb{R}^{N}} Q_{p}^{*}(f) d x: \mathbf{G}_{\alpha p} * f \geq \chi_{E}, f \geq 0, Q_{p}^{*}(f) \in L^{1}\left(\mathbb{R}^{N}\right)\right\},
$$

and

$$
\operatorname{Cap}_{\mathbf{I}_{\alpha p}, Q_{p}^{*}}(E)=\inf \left\{\int_{\mathbb{R}^{N}} Q_{p}^{*}(f) d x: \mathbf{I}_{\alpha p} * f \geq \chi_{E}, f \geq 0, Q_{p}^{*}(f) \in L^{1}\left(\mathbb{R}^{N}\right)\right\},
$$

where $\mathbf{G}_{\alpha p}(x)=\mathcal{F}^{-1}\left(\left(1+|.|^{2}\right)^{-\frac{\alpha p}{2}}\right)(x)$ is the Bessel kernel of order $p$ and $I_{\alpha p}(x)=(N-$ $\alpha p)^{-1}|x|^{-(N-\alpha p)}$.

The expressions $a \wedge b$ and $a \vee b$ stand for $\min \{a, b\}$ and $\max \{a, b\}$ respectively. We denote by $B_{r}$ the ball of center 0 and radius $r>0$. Our main results are the following theorems.

Theorem 1.1 Let $1<p<N, a>0, l \in \mathbb{N}^{*}$ and $\beta \geq 1$ such that $l \beta>p-1$. Let $\Omega \subset \mathbb{R}^{N}$ be $a$ bounded domain. If $\mu$ is a nonnegative Radon measure in $\Omega$, there exists $M>0$ depending on $N, p, l, a, \beta$ and diam $(\Omega)$ (the diameter of $\Omega$ ) such that if

$$
\left\|\mathbf{M}_{p, 2 \operatorname{diam}(\Omega)}^{\frac{(p-1)(\beta-1)}{\beta}}[\mu]\right\|_{L^{\infty}\left(\mathbb{R}^{N}\right)} \leq M,
$$

and $\omega=M\left\|\mathbf{M}_{p, 2 \operatorname{diam}(\Omega)}^{\frac{(p-1)(\beta-1)}{\beta}}[1]\right\|_{L^{\infty}\left(\mathbb{R}^{N}\right)}^{-1}+\mu$ with $c_{p}=1 \vee 4^{\frac{2-p}{p-1}}$, then $P_{l, a, \beta}\left(2 c_{p} K_{1} \mathbf{W}_{1, p}^{2 \operatorname{diam}(\Omega)}[\omega]\right)$ is integrable in $\Omega$ and the following Dirichlet problem

$$
\begin{aligned}
-\Delta_{p} u & =P_{l, a, \beta}(u)+\mu & & \text { in } \Omega, \\
u & =0 & & \text { on } \partial \Omega,
\end{aligned}
$$

admits a nonnegative renormalized solution $u$ which satisfies

$$
u(x) \leq 2 c_{p} K_{1} \mathbf{W}_{1, p}^{2 \operatorname{diam}(\Omega)}[\omega](x) \quad \forall x \in \Omega .
$$

The role of $K_{1}=K_{1}(N, p)$ will be made explicit in Theorem 3.4.

Conversely, if (1.16) admits a nonnegative renormalized solution $u$ and $P_{l, a, \beta}(u)$ is integrable in $\Omega$, then for any compact set $K \subset \Omega$, there exists a positive constant $C$ depending on $N, p, l, a, \beta$ and dist $(K, \partial \Omega)$ such that

$$
\int_{E} P_{l, a, \beta}(u) d x+\mu(E) \leq C C a p_{\mathbf{G}_{p}, Q_{p}^{*}}(E) \quad \text { for all Borel sets } E \subset K .
$$

Furthermore, $u \in W_{0}^{1, p_{1}}(\Omega)$ for all $1 \leq p_{1}<p$.

When $\Omega=\mathbb{R}^{N}$, we have a similar result provided $\mu$ has compact suppport.

Theorem 1.2 Let $1<p<N, a>0, l \in \mathbb{N}^{*}$ and $\beta \geq 1$ such that $l \beta>\frac{N(p-1)}{N-p}$ and $R>0$. If $\mu$ is a nonnegative Radon measure in $\mathbb{R}^{N}$ with $\operatorname{supp}(\mu) \subset B_{R}$ there exists $M>0$ depending on $N, p, l, a, \beta$ and $R$ such that if

$$
\left\|\mathbf{M}_{p}^{\frac{(p-1)(\beta-1)}{\beta}}[\mu]\right\|_{L^{\infty}\left(\mathbb{R}^{N}\right)} \leq M,
$$


and $\omega=M\left\|\mathbf{M}_{p}^{\frac{(p-1)(\beta-1)}{\beta}}\left[\chi_{B_{R}}\right]\right\|_{L^{\infty}\left(\mathbb{R}^{N}\right)}^{-1} \chi_{B_{R}}+\mu$, then $P_{l, a, \beta}\left(2 c_{p} K_{1} \mathbf{W}_{1, p}[\omega]\right)$ is integrable in $\mathbb{R}^{N}$ and the following problem

$$
\begin{aligned}
-\Delta_{p} u & =P_{l, a, \beta}(u)+\mu \text { in } \mathcal{D}^{\prime}\left(\mathbb{R}^{N}\right), \\
\inf _{\mathbb{R}^{N}} u & =0,
\end{aligned}
$$

admits a p-superharmonic solution $u$ which satisfies

$$
u(x) \leq 2 c_{p} K_{1} \mathbf{W}_{1, p}[\omega](x) \quad \forall x \in \mathbb{R}^{N},
$$

( $c_{p}$ and $K_{1}$ as in Theorem 1.1).

Conversely, if (1.19) has a solution $u$ and $P_{l, a, \beta}(u)$ is locally integrable in $\mathbb{R}^{N}$, then there exists a positive constant $C$ depending on $N, p, l, a, \beta$ such that

$$
\int_{E} P_{l, a, \beta}(u) d x+\mu(E) \leq C C a p_{\mathbf{I}_{p}, Q_{p}^{*}}(E) \quad \forall E \subset \mathbb{R}^{N}, E \text { Borel. }
$$

Furthermore, $u \in W_{l o c}^{1, p_{1}}\left(\mathbb{R}^{N}\right)$ for all $1 \leq p_{1}<p$.

Concerning the $k$-Hessian operator we recall some notions introduced by Trudinger and Wang [24, 25, 26], and we follow their notations. For $k=1, \ldots, N$ and $u \in C^{2}(\Omega)$ the k-Hessian operator $F_{k}$ is defined by

$$
F_{k}[u]=S_{k}\left(\lambda\left(D^{2} u\right)\right)
$$

where $\lambda\left(D^{2} u\right)=\lambda=\left(\lambda_{1}, \lambda_{2}, \ldots, \lambda_{N}\right)$ denotes the eigenvalues of the Hessian matrix of second partial derivatives $D^{2} u$ and $S_{k}$ is the k-th elementary symmetric polynomial that is

$$
S_{k}(\lambda)=\sum_{1 \leq i_{1}<\ldots<i_{k} \leq N} \lambda_{i_{1} \ldots \lambda_{i_{k}}}
$$

It is straightforward that

$$
F_{k}[u]=\left[D^{2} u\right]_{k},
$$

where in general $[A]_{k}$ denotes the sum of the k-th principal minors of a matrix $A=\left(a_{i j}\right)$. In order that there exists a smooth k-admissible function which vanishes on $\partial \Omega$, the boundary $\partial \Omega$ must satisfy a uniformly ( $\mathrm{k}-1)$-convex condition, that is

$$
S_{k-1}(\kappa) \geq c_{0}>0 \text { on } \partial \Omega,
$$

for some positive constant $c_{0}$, where $\kappa=\left(\kappa_{1}, \kappa_{2}, \ldots, \kappa_{n-1}\right)$ denote the principal curvatures of $\partial \Omega$ with respect to its inner normal. We also denote by $\Phi^{k}(\Omega)$ the class of upper-semicontinuous functions $\Omega \rightarrow[-\infty, \infty)$ which are $k$-convex, or subharmonic in the Perron sense (see Definition 5.1). In this paper we prove the following theorem (in which expression $\mathbb{E}[q]$ is the largest integer less or equal to $q$ )

Theorem 1.3 Let $k \in\{1,2, \ldots, \mathbb{E}[N / 2]\}$ such that $2 k<N, l \in \mathbb{N}^{*}, \beta \geq 1$ such that $l \beta>k$ and $a>0$. Let $\Omega$ be a bounded uniformly (k-1)-convex domain in $\mathbb{R}^{N}$. Let $\varphi$ be a nonnegative continuous function on $\partial \Omega$ and $\mu=\mu_{1}+f$ be a nonnegative Radon measure where $\mu_{1}$ has compact support in $\Omega$ and $f \in L^{q}(\Omega)$ for some $q>\frac{N}{2 k}$. Let $K_{2}=K_{2}(N, k)$ be the constant $K_{2}$ 
which appears in Theorem 5.3. Then, there exist positive constants $b, M_{1}$ and $M_{2}$ depending on $N, k, l, a, \beta$ and diam $(\Omega)$ such that, if $\max _{\partial \Omega} \varphi \leq M_{2}$ and

$$
\left\|\mathbf{M}_{2 k, 2 \operatorname{diam}(\Omega)}^{\frac{k(\beta-1)}{\beta}}[\mu]\right\|_{L^{\infty}\left(\mathbb{R}^{N}\right)} \leq M_{1},
$$

then $P_{l, a, \beta}\left(2 K_{2} \mathbf{W}_{\frac{2 k}{k+1}, k+1}^{2 \operatorname{diam}(\Omega)}[\mu]+b\right)$ is integrable in $\Omega$ and the following Dirichlet problem

$$
\begin{aligned}
F_{k}[-u] & =P_{l, a, \beta}(u)+\mu & & \text { in } \Omega, \\
u & =\varphi & & \text { texton } \partial \Omega,
\end{aligned}
$$

admits a nonnegative solution $u$, continuous near $\partial \Omega$, with $-u \in \Phi^{k}(\Omega)$ which satisfies

$$
u(x) \leq 2 K_{2} \mathbf{W}_{\frac{2 k}{k+1}, k+1}^{2 \operatorname{diam}(\Omega)}[\mu](x)+b \quad \forall x \in \Omega .
$$

Conversely, if (1.22) admits a nonnegative solution $u$, continuous near $\partial \Omega$, such that $-u \in$ $\Phi^{k}(\Omega)$ and $P_{l, a, \beta}(u)$ is integrable in $\Omega$, then for any compact set $K \subset \Omega$, there exists a positive constant $C$ depending on $N, k, l, a, \beta$ and dist $(K, \partial \Omega)$ such that there holds

$$
\int_{E} P_{l, a, \beta}(u) d x+\mu(E) \leq C C a p_{\mathbf{G}_{2 k}, Q_{k+1}^{*}}(E) \quad \forall E \subset K, E \text { Borel, }
$$

where $Q_{k+1}(s)$ is defined by (1.13) with $p=k+1, Q_{k+1}^{*}$ is its complementary function and $\mathrm{Cap}_{\mathbf{G}_{2 k}, Q_{k+1}^{*}}(E)$ is defined accordingly by (1.14).

The following extension holds when $\Omega=\mathbb{R}^{N}$.

Theorem 1.4 Let $k \in\{1,2, \ldots, \mathbb{E}[N / 2]\}$ such that $2 k<N, l \in \mathbb{N}^{*}, \beta \geq 1$ such that $l \beta>\frac{N k}{N-2 k}$ and $a>0, R>0$. If $\mu$ is a nonnegative Radon measure in $\mathbb{R}^{N}$ with $\operatorname{supp}(\mu) \subset B_{R}$ there exists $M>0$ depending on $N, k, l, a, \beta$ and $R$ such that if

$$
\left\|\mathbf{M}_{2 k}^{\frac{k(\beta-1)}{\beta}}[\mu]\right\|_{L^{\infty}\left(\mathbb{R}^{N}\right)} \leq M,
$$

and $\omega=M\left\|\mathbf{M}_{2 k}^{\frac{k(\beta-1)}{\beta}}\left[\chi_{B_{R}}\right]\right\|_{L^{\infty}\left(\mathbb{R}^{N}\right)}^{-1} \chi_{B_{R}}+\mu$, then $P_{l, a, \beta}\left(2 K_{2} \mathbf{W}_{\frac{2 k}{k+1}, k+1}[\omega]\right)$ is integrable in $\mathbb{R}^{N}$ $\left(K_{2}\right.$ as in Theorem 1.3) and the following Dirichlet problem

$$
\begin{aligned}
F_{k}[-u] & =P_{l, a, \beta}(u)+\mu \text { in } \mathbb{R}^{N}, \\
\inf _{\mathbb{R}^{N}} u & =0,
\end{aligned}
$$

admits a nonnegative solution $u$ with $-u \in \Phi^{k}\left(\mathbb{R}^{N}\right)$ which satisfies

$$
u(x) \leq 2 K_{2} \mathbf{W}_{\frac{2 k}{k+1}, k+1}[\omega](x) \quad \forall x \in \mathbb{R}^{N} .
$$

Conversely, if (1.25) admits a nonnegative solution $u$ with $-u \in \Phi^{k}\left(\mathbb{R}^{N}\right)$ and $P_{l, a, \beta}(u)$ locally integrable in $\mathbb{R}^{N}$, then there exists a positive constant $C$ depending on $N, k, l, a, \beta$ such that there holds

$$
\int_{E} P_{l, a, \beta}(u) d x+\mu(E) \leq C \operatorname{Cap}_{\mathbf{I}_{2 k}, Q_{k+1}^{*}}(E) \quad \forall E \subset \mathbb{R}^{N}, E \text { Borel. }
$$

where $\operatorname{Cap}_{\mathbf{I}_{2 k}, Q_{k+1}^{*}}(E)$ is defined accordingly by (1.15). 
The four previous theorems are connected to the following results which deals with a class of nonlinear Wolff integral equations.

Theorem 1.5 Let $\alpha>0, p>1, a>0, \varepsilon>0, R>0, l \in \mathbb{N}^{*}$ and $\beta \geq 1$ such that $l \beta>p-1$ and $0<\alpha p<N$. Let $f$ be a nonnegative measurable in $\mathbb{R}^{N}$ with the property that $\mu_{1}=P_{l, a+\varepsilon, \beta}(f)$ is locally integrable in $\mathbb{R}^{N}$ and $\mu \in \mathfrak{M}^{+}\left(\mathbb{R}^{N}\right)$. There exists $M>0$ depending on $N, \alpha, p, l, a, \beta, \varepsilon$ and $R$ such that if

$$
\left\|\mathbf{M}_{\alpha p, R}^{\frac{(p-1)(\beta-1)}{\beta}}[\mu]\right\|_{L^{\infty}\left(\mathbb{R}^{N}\right)} \leq M \quad \text { and } \quad\left\|\mathbf{M}_{\alpha p, R}^{\frac{(p-1)(\beta-1)}{\beta}}\left[\mu_{1}\right]\right\|_{L^{\infty}\left(\mathbb{R}^{N}\right)} \leq M
$$

then there exists a nonnegative function $u$ such that $P_{l, a, \beta}(u)$ is locally integrable in $\mathbb{R}^{N}$ which satisfies

$$
u=\mathbf{W}_{\alpha, p}^{R}\left[P_{l, a, \beta}(u)+\mu\right]+f \text { in } \mathbb{R}^{N},
$$

and

$$
u \leq F:=2 c_{p} \mathbf{W}_{\alpha, p}^{R}\left[\omega_{1}\right]+2 c_{p} \mathbf{W}_{\alpha, p}^{R}\left[\omega_{2}\right]+f, \quad P_{l, a, \beta}(F) \in L_{l o c}^{1}\left(\mathbb{R}^{N}\right),
$$

where $\omega_{1}=\left.M\left\|\mathbf{M}_{\alpha p, R}^{\frac{(p-1)(\beta-1)}{\beta}}[1]\right\|\right|_{L^{\infty}\left(\mathbb{R}^{N}\right)} ^{-1}+\mu$ and $\omega_{2}=M\left\|\mathbf{M}_{\alpha p, R}^{\frac{(p-1)(\beta-1)}{\beta}}[1]\right\|_{L^{\infty}\left(\mathbb{R}^{N}\right)}^{-1}+\mu_{1}$.

Conversely, if (1.29) admits a nonnegative solution $u$ and $P_{l, a, \beta}(u)$ is locally integrable in $\mathbb{R}^{N}$, then there exists a positive constant $C$ depending on $N, \alpha, p, l, a, \beta$ and $R$ such that there holds

$$
\int_{E} P_{l, a, \beta}(u) d x+\int_{E} P_{l, a+\varepsilon, \beta}(f) d x+\mu(E) \leq C C_{a p} P_{\mathbf{G}_{\alpha p}, Q_{p}^{*}}(E) \quad \forall E \subset \mathbb{R}^{N}, E \text { Borel. }
$$

When $R=\infty$ in the above theorem, we have a similar result provided $f$ and $\mu$ have compact support in $\mathbb{R}^{N}$.

Theorem 1.6 Let $\alpha>0, p>1, a>0, \varepsilon>0, R>0, l \in \mathbb{N}^{*}$ and $\beta \geq 1$ such that $0<\alpha p<N$ and $l \beta>\frac{N(p-1)}{N-\alpha p}$. There exists $M>0$ depending on $N, \alpha, p, l, a, \beta, \varepsilon$ and $R$ such that if $f$ is a nonnegative measurable function in $\mathbb{R}^{N}$ with support in $B_{R}$ such that $\mu_{1}=P_{l, a+\varepsilon, \beta}(f)$ is locally integrable in $\mathbb{R}^{N}$ and $\mu$ is a positive measure in $\mathbb{R}^{N}$ with support in $B_{R}$ which verify

$$
\left\|\mathbf{M}_{\alpha p}^{\frac{(p-1)(\beta-1)}{\beta}}[\mu]\right\|_{L^{\infty}\left(\mathbb{R}^{N}\right)} \leq M \quad \text { and } \quad\left\|\mathbf{M}_{\alpha p}^{\frac{(p-1)(\beta-1)}{\beta}}\left[\mu_{1}\right]\right\|_{L^{\infty}\left(\mathbb{R}^{N}\right)} \leq M
$$

then there exists a nonnegative function $u$ such that $P_{l, a, \beta}(u)$ is integrable in $\mathbb{R}^{N}$ which satisfies

$$
u=\mathbf{W}_{\alpha, p}\left[P_{l, a, \beta}(u)+\mu\right]+f \text { in } \mathbb{R}^{N},
$$

and

$$
u \leq F:=2 c_{p} \mathbf{W}_{\alpha, p}\left[\omega_{1}\right]+2 c_{p} \mathbf{W}_{\alpha, p}\left[\omega_{2}\right]+f, \quad P_{l, a, \beta}(F) \in L^{1}\left(\mathbb{R}^{N}\right),
$$

where $\omega_{1}=M\left\|\mathbf{M}_{\alpha p}^{\frac{(p-1)(\beta-1)}{\beta}}\left[\chi_{B_{R}}\right]\right\|_{L^{\infty}\left(\mathbb{R}^{N}\right)}^{-1} \chi_{B_{R}}+\mu$ and $\omega_{2}=M\left\|\mathbf{M}_{\alpha p}^{\frac{(p-1)(\beta-1)}{\beta}}\left[\chi_{B_{R}}\right]\right\|_{L^{\infty}\left(\mathbb{R}^{N}\right)}^{-1} \chi_{B_{R}}+$ $\mu_{1}$.

Conversely, if (1.33) admits a nonnegative solution u such that $P_{l, a, \beta}(u)$ is integrable in $\mathbb{R}^{N}$, then there exists a positive constant $C$ depending on $N, \alpha, p, l, a, \beta$ such that there holds

$$
\int_{E} P_{l, a, \beta}(u) d x+\int_{E} P_{l, a, \beta}(f) d x+\mu(E) \leq C \operatorname{Cap}_{\mathbf{I}_{\alpha p}, Q_{p}^{*}}(E) \quad \forall E \subset \mathbb{R}^{N}, E \text { Borel. }
$$


As an application of the Wolff integral equation we can notice that $\alpha=1$, equation (1.33) is equivalent to

$$
-\Delta_{p}(u-f)=P_{l, a, \beta}(u)+\mu \quad \text { in } \mathbb{R}^{N} .
$$

When $\alpha=\frac{2 k}{k+1}$ and $p=k+1$, it is equivalent to

$$
F_{k}[-u+f]=P_{l, a, \beta}(u)+\mu \quad \text { in } \mathbb{R}^{N} .
$$

If $p=2$ equation (1.33) becomes linear. If we set $\gamma=2 \alpha$, then

$$
\begin{aligned}
\mathbf{W}_{\alpha, 2}[\omega](x) & =\int_{0}^{\infty} \omega\left(B_{t}(x)\right) \frac{d t}{t^{N-\gamma+1}} \\
& =\int_{\mathbb{R}^{N}}\left(\int_{|x-y|}^{\infty} \frac{d t}{t^{N-\gamma+1}}\right) d \mu(y) \\
& =\frac{1}{N-\gamma} \int_{\mathbb{R}^{N}} \frac{d \omega(y)}{|x-y|^{N-\gamma}} \\
& =I_{\gamma} * \omega,
\end{aligned}
$$

where $I_{\gamma}$ is the Riesz kernel of order $\gamma$. Thus (1.33) is equivalent to

$$
(-\Delta)^{\alpha}(u-f)=P_{l, a, \beta}(u)+\mu \quad \text { in } \mathbb{R}^{N} .
$$

Remark 1.7 In case $\Omega$ is a bounded open set, uniformly bounded of sequence $\left\{u_{n}\right\}$ (2.22) is essential for the existence of solutions of equations (1.16), (1.22) and (1.29). Moreover, conditions $l \beta>p-1$ in Theorem 1.1, 1.5 and $l \beta>k$ in Theorem 1.3 is necessary so as to get (2.22) from iteration schemes (2.27). Besides, in case $\Omega=\mathbb{R}^{N}$, equation (1.19) in Theorem 1.2 ( (1.25) in Theorem 1.4. (1.33) in Theorem 1.6 resp.) has nontrivial solution on $\mathbb{R}^{N}$ if and only if $l \beta>\frac{N(p-1)}{N-p}\left(l \beta>\frac{N k}{N-2 k}, l \beta>\frac{N(p-1)}{N-\alpha p}\right.$ resp. $)$. In fact, here we only need to consider equation (1.19). Assume that $l \beta \leq \frac{N(p-1)}{N-p}$, using Holder inequality we have $P_{l, a, \beta}(u) \geq c u^{\gamma}$ where $p-1<\gamma \leq \frac{N(p-1)}{N-p}$, so we get from Theorem (3.4).

$$
u \geq K \mathbf{W}_{1, p}\left[c u^{\gamma}+\mu\right] \quad \text { in } \mathbb{R}^{N}
$$

for some constant $K$. Therefore, we can verify that

$$
\int_{E} u^{\gamma} d x+\mu(E) \leq C \operatorname{Cap}_{\mathbf{I}_{p}, \frac{\gamma}{\gamma-p+1}}(E) \quad \forall E \subset \mathbb{R}^{N}, E \text { Borel. }
$$

see Theorem 2.7. where $C$ is a constant and Cap $\mathbf{I}_{p, \frac{\gamma}{\gamma-p+1}}$ is a Riesz capacity.

Since $N \leq \frac{p \gamma}{\gamma-p+1}\left(\Leftrightarrow p-1<\gamma \leq \frac{N(p-1)}{N-p}\right), \operatorname{Cap}_{\mathbf{I}_{p}, \frac{\gamma}{\gamma-p+1}}(E)=0$ for all Borel set $E$, see [1]. Immediately, we deduce $u \equiv 0$ and $\mu \equiv 0$. 


\section{Estimates on potentials and Wolff integral equations}

We denote by $B_{r}(a)$ the ball of center $a$ and radius $r>0, B_{r}=B_{r}(0)$ and by $\chi_{E}$ the characteristic function of a set $E$. The next estimates are crucial in the sequel.

Theorem 2.1 Let $\alpha>0, p>1$ such that $0<\alpha p<N$.

1. There exists a positive constant $c_{1}$, depending only on $N, \alpha, p$ such that for all $\mu \in \mathfrak{M}^{+}\left(\mathbb{R}^{N}\right)$ and $q \geq p-1,0<R \leq \infty$ we have

$$
\left(c_{1} q\right)^{-\frac{q}{p-1}} \int_{\mathbb{R}^{N}}\left(\mathbf{I}_{\alpha p}^{R}[\mu](x)\right)^{\frac{q}{p-1}} d x \leq \int_{\mathbb{R}^{N}}\left(\mathbf{W}_{\alpha, p}^{R}[\mu](x)\right)^{q} d x \leq\left(c_{1} q\right)^{q} \int_{\mathbb{R}^{N}}\left(\mathbf{I}_{\alpha p}^{R}[\mu](x)\right)^{\frac{q}{p-1}} d x,
$$

2. Let $R>0$. There exists a positive constant $c_{2}$, depending only on $N, \alpha, p, R$ such that for all $\mu \in \mathfrak{M}^{+}\left(\mathbb{R}^{N}\right)$ and $q \geq p-1$ we have

$$
\left(c_{2} q\right)^{-\frac{q}{p-1}} \int_{\mathbb{R}^{N}}\left(\mathbf{G}_{\alpha p}[\mu](x)\right)^{\frac{q}{p-1}} d x \leq \int_{\mathbb{R}^{N}}\left(\mathbf{W}_{\alpha, p}^{R}[\mu](x)\right)^{q} d x \leq\left(c_{2} q\right)^{q} \int_{\mathbb{R}^{N}}\left(\mathbf{G}_{\alpha p}[\mu](x)\right)^{\frac{q}{p-1}} d x,
$$

where $\mathbf{G}_{\alpha p}[\mu]:=\mathbf{G}_{\alpha p} * \mu$ denotes the Bessel potential of order $\alpha p$ of $\mu$.

3. There exists a positive constant $c_{3}$, depending only on $N, \alpha, R$ such that for all $\mu \in \mathfrak{M}^{+}\left(\mathbb{R}^{N}\right)$ and $q \geq 1$ we have

$$
c_{3}^{-q} \int_{\mathbb{R}^{N}}\left(\mathbf{G}_{\alpha}[\mu](x)\right)^{q} d x \leq \int_{\mathbb{R}^{N}}\left(\mathbf{I}_{\alpha}^{R}[\mu](x)\right)^{q} d x \leq c_{3}^{q} \int_{\mathbb{R}^{N}}\left(\mathbf{G}_{\alpha}[\mu](x)\right)^{q} d x .
$$

Proof. Note that $W_{\frac{\alpha}{2}, 2}^{R}[\mu]=I_{\alpha}^{R}[\mu]$. We can find proof of (2.3) in [8, Step 3, Theorem 2.3]. By [8. Step 2, Theorem 2.3], there is $c_{4}>0$ such that

$$
\int_{\mathbb{R}^{N}}\left(\mathbf{W}_{\alpha, p}^{R}[\mu](x)\right)^{q} d x \geq c_{4}^{q} \int_{\mathbb{R}^{N}}\left(\mathbf{M}_{\alpha p, R}[\mu](x)\right)^{\frac{q}{p-1}} d x \forall q \geq p-1,0<R \leq \infty \text { and } \mu \in \mathfrak{M}^{+}\left(\mathbb{R}^{N}\right) .
$$

We recall that $\mathbf{M}_{\alpha p, R}[\mu]=\mathbf{M}_{\alpha p, R}^{0}[\mu]$ by (1.10). Next we show that for all $q \geq p-1,0<R \leq \infty$ and $\mu \in \mathfrak{M}^{+}\left(\mathbb{R}^{N}\right)$ there holds

$$
\int_{\mathbb{R}^{N}}\left(\mathbf{M}_{\alpha p, R}[\mu](x)\right)^{\frac{q}{p-1}} d x \geq\left(c_{5} q\right)^{-q} \int_{\mathbb{R}^{N}}\left(\mathbf{W}_{\alpha, p}^{R}[\mu](x)\right)^{q} d x,
$$

for some positive constant $c_{5}$ depending on $N, \alpha, p$. Indeed, we denote $\mu_{n}$ by $\chi_{B_{n}} \mu$ for $n \in \mathbb{N}^{*}$. By [17, Theorem 1.2] or [8, Proposition 2.2], there exist constants $c_{6}=c_{6}(N, \alpha, p)>0, a=$ $a(\alpha, p)>0$ and $\varepsilon_{0}=\varepsilon(N, \alpha, p)$ such that for all $n \in \mathbb{N}^{*}, t>0,0<R \leq \infty$ and $0<\varepsilon<\varepsilon_{0}$, there holds

$$
\left|\left\{\mathbf{W}_{\alpha, p}^{R} \mu_{n}>3 t\right\}\right| \leq c_{6} \exp \left(-a \varepsilon^{-1}\right)\left|\left\{\mathbf{W}_{\alpha, p}^{R} \mu_{n}>t\right\}\right|+\left|\left\{\left(\mathbf{M}_{\alpha p, R} \mu_{n}\right)^{\frac{1}{p-1}}>\varepsilon t\right\}\right| .
$$

Multiplying by $q t^{q-1}$ and integrating over $(0, \infty)$, we obtain

$$
\begin{aligned}
\int_{0}^{\infty} q t^{q-1}\left|\left\{\mathbf{W}_{\alpha, p}^{R} \mu_{n}>3 t\right\}\right| d t \leq c_{6} \exp \left(-a \varepsilon^{-1}\right) & \int_{0}^{\infty} q t^{q-1}\left|\left\{\mathbf{W}_{\alpha, p}^{R} \mu_{n}>t\right\}\right| d t \\
& +\int_{0}^{\infty} q t^{q-1}\left|\left\{\left(\mathbf{M}_{\alpha p, R} \mu_{n}\right)^{\frac{1}{p-1}}>\varepsilon t\right\}\right| d t
\end{aligned}
$$


which implies

$$
\varepsilon^{q}\left(3^{-q}-c_{6} \exp \left(-a \varepsilon^{-1}\right)\right) \int_{\mathbb{R}^{N}}\left(\mathbf{W}_{\alpha, p}^{R}\left[\mu_{n}\right](x)\right)^{q} d x \leq \int_{\mathbb{R}^{N}}\left(\mathbf{M}_{\alpha p, R} \mu_{n}\right)^{\frac{q}{p-1}} d x .
$$

We see that $\sup _{0<\varepsilon<\varepsilon_{0}} \varepsilon^{q}\left(3^{-q}-c_{6} \exp \left(-a \varepsilon^{-1}\right)\right) \geq\left(c_{7} q\right)^{-q}$ for some constant $c_{7}$ which does not depend on $q$. Therefore (2.5) follows by Fatou's lemma. Hence, it is easy to obtain (2.1) from (2.4) and (2.5). At end, we obtain (2.2) from (2.1) and (2.3).

The next result is proved in 8 .

Theorem 2.2 Let $\alpha>0, p>1,0 \leq \eta<p-1,0<\alpha p<N$ and $L>0$. Set $\delta=$ $\frac{1}{2}\left(\frac{p-1-\eta}{12(p-1)}\right)^{\frac{p-1}{p-1-\eta}} \alpha p \log (2)$. Then there exists $C(L)>0$, depending on $N, \alpha, p, \eta$ and $L$ such that for any $R \in(0, \infty], \mu \in \mathfrak{M}^{+}\left(\mathbb{R}^{N}\right)$, any $a \in \mathbb{R}^{N}$ and $0<r \leq L$, there holds

$$
\frac{1}{\left|B_{2 r}(a)\right|} \int_{B_{2 r}(a)} \exp \left(\delta \frac{\left(\mathbf{W}_{\alpha, p}^{R}\left[\mu_{B_{r}(a)}\right](x)\right)^{\frac{p-1}{p-1-\eta}}}{\|\left.\mathbf{M}_{\alpha p, R}^{\eta}\left[\mu_{B_{r}(a)}\right]\right|_{L^{\infty}\left(B_{r}(a)\right)} ^{\frac{1}{p-1}}}\right) d x \leq C(L),
$$

where $\mu_{B_{r}(a)}=\chi_{B_{r}(a)} \mu$. Furthermore, if $\eta=0, C$ is independent of $L$.

Theorem 2.3 Let $\alpha>0, p>1$ with $0<\alpha p<N, \beta \geq 1$ and $R>0$. Assume $\mu \in \mathfrak{M}^{+}\left(\mathbb{R}^{N}\right)$ satisfies

$$
\left\|\mathbf{M}_{\alpha p, R}^{\frac{(p-1)(\beta-1)}{\beta}}[\mu]\right\|_{L^{\infty}\left(\mathbb{R}^{N}\right)} \leq 1,
$$

and set $\omega=\left\|\mathbf{M}_{\alpha p, R}^{\frac{(p-1)(\beta-1)}{\beta}}[1]\right\|_{L^{\infty}\left(\mathbb{R}^{N}\right)}^{-1}+\mu$. Then there exist positive constants $C, \delta_{0}$ and $c$ independent on $\mu$ such that $\exp \left(\delta_{0}\left(\mathbf{W}_{\alpha, p}^{R}[\omega]\right)^{\beta}\right)$ is locally integrable in $\mathbb{R}^{N}$,

$$
\left\|\mathbf{W}_{\alpha, p}^{R}\left[\exp \left(\delta_{0}\left(\mathbf{W}_{\alpha, p}^{R}[\omega]\right)^{\beta}\right)\right]\right\|_{L^{\infty}\left(\mathbb{R}^{N}\right)} \leq C
$$

and

$$
\mathbf{W}_{\alpha, p}^{R}\left[\exp \left(\delta_{0}\left(\mathbf{W}_{\alpha, p}^{R}[\omega]\right)^{\beta}\right)\right] \leq c \mathbf{W}_{\alpha, p}^{R}[\omega] \quad \text { in } \mathbb{R}^{N} .
$$

Proof. Let $\delta$ be as in Theorem 2.2, From (2.7), we have

$$
\left\|\mathbf{M}_{\alpha p, R}^{\frac{(p-1)(\beta-1)}{\beta}}[\omega]\right\|_{L^{\infty}\left(\mathbb{R}^{N}\right)} \leq 2 .
$$

Let $x \in \mathbb{R}^{N}$. Since $\omega\left(B_{t}(y)\right) \leq 2 t^{N-\alpha p} h_{\frac{(p-1)(\beta-1)}{\beta}}(t)$, for all $r \in(0, R)$ and $y \in \mathbb{R}^{N}$ we have

$$
\begin{aligned}
\mathbf{W}_{\alpha, p}^{R}[\omega](y) & =\mathbf{W}_{\alpha, p}^{r}[\omega](y)+\int_{r}^{R}\left(\frac{\omega\left(B_{t}(y)\right)}{t^{N-\alpha p}}\right)^{\frac{1}{p-1}} \frac{d t}{t} \\
& \leq \mathbf{W}_{\alpha, p}^{r}[\omega](y)+2^{\frac{1}{p-1}} \int_{r \wedge 2^{-1}}^{2^{-1}}(-\ln t)^{-\frac{\beta-1}{\beta}} \frac{d t}{t}+2^{\frac{1}{p-1}} \int_{2^{-1}}^{R \vee 2^{-1}}(-\ln t)^{-\frac{\beta-1}{\beta}} \frac{d t}{t} \\
& \leq \mathbf{W}_{\alpha, p}^{r}[\omega](y)+c_{8}\left(-\ln \left(r \wedge 2^{-1}\right)\right)^{\frac{1}{\beta}}+c_{8} .
\end{aligned}
$$


Thus,

$$
\left(\mathbf{W}_{\alpha, p}^{R}[\omega](y)\right)^{\beta} \leq 3^{\beta-1}\left(\mathbf{W}_{\alpha, p}^{r}[\omega](y)\right)^{\beta}+c_{9} \ln \left(\frac{1}{r \wedge 2^{-1}}\right)+c_{9} .
$$

Let $\theta \in\left(0,2^{-\frac{\beta}{p-1}}\right]$, since $\exp \left(\frac{a+b}{2}\right) \leq \exp (a)+\exp (b)$ for all $a, b \in \mathbb{R}$, we get from (2.10)

$$
\begin{aligned}
\exp \left(\theta \delta 3^{-\beta}\left(\mathbf{W}_{\alpha, p}^{R}[\omega](y)\right)^{\beta}\right) & \leq \exp \left(\delta 2^{-\frac{\beta}{p-1}}\left(\mathbf{W}_{\alpha, p}^{r}[\omega](y)\right)^{\beta}\right)+c_{10} \exp \left(\theta c_{11} \ln \left(\frac{1}{r \wedge 2^{-1}}\right)\right) \\
& =\exp \left(\delta 2^{-\frac{\beta}{p-1}}\left(\mathbf{W}_{\alpha, p}^{r}[\omega](y)\right)^{\beta}\right)+c_{10}\left(r \wedge 2^{-1}\right)^{-\theta c_{11}} .
\end{aligned}
$$

For $r>0,0<t \leq r, y \in B_{r}(x)$ there holds $B_{t}(y) \subset B_{2 r}(x)$. Thus, $\mathbf{W}_{\alpha, p}^{r}[\omega]=\mathbf{W}_{\alpha, p}^{r}\left[\omega_{B_{2 r}(x)}\right]$ in $B_{r}(x)$. Then, using (2.6) in Theorem 2.2 with $\eta=\frac{(p-1)(\beta-1)}{\beta}$ and $L=2 R$ we get

$$
\int_{B_{r}(x)} \exp \left(\delta 2^{-\frac{\beta}{p-1}}\left(\mathbf{W}_{\alpha, p}^{r}[\omega]\right)^{\gamma}\right)=\int_{B_{r}(x)} \exp \left(\delta 2^{-\frac{\beta}{p-1}}\left(\mathbf{W}_{\alpha, p}^{r}\left[\omega_{B_{2 r}(x)}\right]\right)^{\gamma}\right) \leq c_{12} r^{N}
$$

Therefore, taking $\theta=2^{-\frac{\beta}{p-1}} \wedge \frac{\alpha p}{2 c_{11}}$, we deduce from (2.11)

$$
\begin{aligned}
\mathbf{W}_{\alpha, p}^{R}\left[\exp \left(\theta \delta 3^{-\beta}\left(\mathbf{W}_{\alpha, p}^{R}[\omega]\right)^{\gamma}\right)\right](x) & \leq \int_{0}^{R}\left(c_{12} r^{\alpha p}+c_{13}\left(r \wedge 2^{-1}\right)^{-\theta c_{11}} r^{\alpha p}\right)^{\frac{1}{p-1}} \frac{d r}{r} \\
& \leq \int_{0}^{R}\left(c_{12} r^{\alpha p}+c_{13}\left(r \wedge 2^{-1}\right)^{-\frac{\alpha p}{2}} r^{\alpha p}\right)^{\frac{1}{p-1}} \frac{d r}{r} \\
& \leq c_{14} .
\end{aligned}
$$

Hence, we get (2.8) with $\delta_{0}=\left(2^{-\frac{\beta}{p-1}} \wedge \frac{\alpha p}{2 c_{11}}\right) \delta 3^{-\beta}$; we also get (2.9) since $\mathbf{W}_{\alpha, p}^{R}[\omega] \geq c_{15}$ for some positive constant $c_{15}>0$.

We recall that $H_{l}$ and $P_{l, a, \beta}$ have been defined in (1.11) and (1.12).

Theorem 2.4 Let $\alpha>0, p>1, l \in \mathbb{N}^{*}$ and $\beta \geq 1$ such that $0<\alpha p<N, l \beta>\frac{N(p-1)}{N-\alpha p}$ and $R>0$. Assume that $\mu \in \mathfrak{M}^{+}\left(\mathbb{R}^{N}\right)$ has support in $B_{R}$ and verifies

$$
\left\|\mathbf{M}_{\alpha p}^{\frac{(p-1)(\beta-1)}{\beta}}[\mu]\right\|_{L^{\infty}\left(\mathbb{R}^{N}\right)} \leq 1
$$

and set $\omega=\left\|\mathbf{M}_{\alpha p}^{\frac{(p-1)(\beta-1)}{\beta}}\left[\chi_{B_{R}}\right]\right\|_{L^{\infty}\left(\mathbb{R}^{N}\right)}^{-1} \chi_{B_{R}}+\mu$. Then there exist $C=C(N, \alpha, p, l, \beta, R)>0$ and $\delta_{1}=\delta_{1}(N, \alpha, p, l, \beta, R)>0$ such that $H_{l}\left(\delta_{1}\left(\mathbf{W}_{\alpha, p}[\omega]\right)^{\beta}\right)$ is integrable in $\mathbb{R}^{N}$ and

$$
\mathbf{W}_{\alpha, p}\left[H_{l}\left(\delta_{1}\left(\mathbf{W}_{\alpha, p}[\omega]\right)^{\beta}\right)\right](x) \leq C \mathbf{W}_{\alpha, p}[\omega](x) \quad \forall x \in \mathbb{R}^{N} .
$$

Proof. We have from (2.12)

$$
\left\|\mathbf{M}_{\alpha p}^{\frac{(p-1)(\beta-1)}{\beta}}[\omega]\right\|_{L^{\infty}\left(\mathbb{R}^{N}\right)} \leq 2 .
$$


In particular, $\omega\left(B_{R}\right) \leq c_{16}$. Let $\delta_{1}>0$ and $x \in \mathbb{R}^{N}$ fixed. We split the Wolff potential $\mathbf{W}_{\alpha, p}[\omega]$ into lower and upper parts defined by

$$
\mathbf{L}_{\alpha, p}^{t}[\omega](x)=\int_{t}^{+\infty}\left(\frac{\omega\left(B_{r}(x)\right)}{r^{N-\alpha p}}\right)^{\frac{1}{p-1}} \frac{d r}{r},
$$

and

$$
\mathbf{W}_{\alpha, p}^{t}[\omega](x)=\int_{0}^{t}\left(\frac{\omega\left(B_{r}(x)\right)}{r^{N-\alpha p}}\right)^{\frac{1}{p-1}} \frac{d r}{r} .
$$

Using the convexity we have

$$
H_{l}\left(\delta_{1}\left(\mathbf{W}_{\alpha, p}[\omega]\right)^{\beta}\right) \leq H_{l}\left(\delta_{1} 2^{\beta}\left(\mathbf{L}_{\alpha, p}^{t}[\omega]\right)^{\beta}\right)+H_{l}\left(\delta_{1} 2^{\beta}\left(\mathbf{W}_{\alpha, p}^{t}[\omega]\right)^{\beta}\right) .
$$

Thus,

$\mathbf{W}_{\alpha, p}\left[H_{l}\left(\delta_{1}\left(\mathbf{W}_{\alpha, p}[\omega]\right)^{\beta}\right)\right](x) \leq c_{17} \int_{0}^{+\infty}\left(\frac{\omega_{t}^{1}\left(B_{t}(x)\right)}{t^{N-\alpha p}}\right)^{\frac{1}{p-1}} \frac{d t}{t}+c_{17} \int_{0}^{+\infty}\left(\frac{\omega_{t}^{2}\left(B_{t}(x)\right)}{t^{N-\alpha p}}\right)^{\frac{1}{p-1}} \frac{d t}{t}$,

where $d \omega_{t}^{1}=H_{l}\left(\delta_{1} 2^{\beta}\left(\mathbf{L}_{\alpha, p}^{t}[\omega]\right)^{\beta}\right) d x$ and $d \omega_{t}^{2}=H_{l}\left(\delta_{1} 2^{\beta}\left(\mathbf{W}_{\alpha, p}^{t}[\omega]\right)^{\beta}\right) d x$. Inequality (2.13) will follows from the two inequalities below,

$$
\int_{0}^{+\infty}\left(\frac{\omega_{t}^{1}\left(B_{t}(x)\right)}{t^{N-\alpha p}}\right)^{\frac{1}{p-1}} \frac{d t}{t} \leq c_{18} \mathbf{W}_{\alpha, p}[\omega](x),
$$

and

$$
\omega_{t}^{2}\left(B_{t}(x)\right) \leq c_{18} \omega\left(B_{4 t}(x)\right) .
$$

Step 1: Proof of (2.15). Since $B_{r}(y) \subset B_{2 r}(x)$ for $y \in B_{t}(x)$ and $r \geq t$, there holds

$$
\mathbf{L}_{\alpha, p}^{t}[\omega](y) \leq \int_{t}^{+\infty}\left(\frac{\omega\left(B_{2 r}(x)\right)}{r^{N-\alpha p}}\right)^{\frac{1}{p-1}} \frac{d r}{r}=2^{\frac{N-\alpha p}{p-1}} \mathbf{L}_{\alpha, p}^{2 t}[\omega](x) .
$$

It follows

$$
\omega_{t}^{1}\left(B_{t}(x)\right) \leq\left|B_{1}(0)\right| t^{N} H_{l}\left(\delta_{1} c_{19}\left(\mathbf{L}_{\alpha, p}^{2 t}[\omega](x)\right)^{\beta}\right) .
$$

Thus,

$$
\int_{0}^{+\infty}\left(\frac{\omega_{t}^{1}\left(B_{t}(x)\right)}{t^{N-\alpha p}}\right)^{\frac{1}{p-1}} \frac{d t}{t} \leq c_{20} \int_{0}^{\infty} A_{t}(x) d t
$$

where

$$
A_{t}(x)=\left(t^{\alpha p} H_{l}\left(\delta_{1} c_{19}\left(\mathbf{L}_{\alpha, p}^{2 t}[\omega](x)\right)^{\beta}\right)\right)^{\frac{1}{p-1}} \frac{1}{t} .
$$

Since $H_{l}(s) \leq s^{l} \exp (s)$ for all $s \geq 0$,

$$
\begin{aligned}
A_{t}(x) & \leq c_{21}\left(t^{\alpha p}\left(\mathbf{L}_{\alpha, p}^{2 t}[\omega](x)\right)^{l \beta} \exp \left(\delta_{1} c_{19}\left(\mathbf{L}_{\alpha, p}^{2 t}[\omega](x)\right)^{\beta}\right)\right)^{\frac{1}{p-1}} \frac{1}{t} \\
& =c_{21} t^{\frac{\alpha p}{p-1}-1}\left(\mathbf{L}_{\alpha, p}^{2 t}[\omega](x)\right)^{\frac{l \beta-p+1}{p-1}} \exp \left(\delta_{1} c_{22}\left(\mathbf{L}_{\alpha, p}^{2 t}[\omega](x)\right)^{\beta}\right) \mathbf{L}_{\alpha, p}^{2 t}[\omega](x) .
\end{aligned}
$$


Now we estimate $\mathbf{L}_{\alpha, p}^{2 t}[\omega]$.

Case 1: $t \in(0,1)$. From (2.14) we deduce

$$
\begin{aligned}
\mathbf{L}_{\alpha, p}^{2 t}[\omega](x) & \leq \int_{t / 2}^{1 / 2}\left(\frac{\omega\left(B_{s}(x)\right)}{s^{N-\alpha p}}\right)^{\frac{1}{p-1}} \frac{d s}{s}+\int_{1 / 2}^{\infty}\left(\frac{\omega\left(B_{s}(x)\right)}{s^{N-\alpha p}}\right)^{\frac{1}{p-1}} \frac{d s}{s} \\
& \leq c_{23} \int_{t / 2}^{1 / 2}(-\ln (s))^{-1+\frac{1}{\beta}} \frac{d s}{s}+\int_{1 / 2}^{\infty}\left(\frac{\omega\left(B_{R}\right)}{s^{N-\alpha p}}\right)^{\frac{1}{p-1}} \frac{d s}{s} \\
& \leq c_{24}(-\ln (t / 2))^{\frac{1}{\beta}},
\end{aligned}
$$

which implies

$$
\begin{aligned}
A_{t}(x) & \leq c_{25} t^{\frac{\alpha p}{p-1}-1}(-\ln (t / 2))^{\frac{l \beta-p+1}{\beta(p-1)}} \exp \left(\delta_{1} c_{26}(-\ln (t / 2))\right) \mathbf{L}_{\alpha, p}^{2 t}[\omega](x) \\
& =c_{27} t^{\frac{\alpha p}{p-1}-1}(-\ln (t / 2))^{\frac{l \beta-p+1}{\beta(p-1)}} t^{-\delta_{1} c_{26}} \mathbf{L}_{\alpha, p}^{2 t}[\omega](x) .
\end{aligned}
$$

We take $\delta_{1} \leq \frac{1}{2 c_{26}}\left(\frac{\alpha p}{p-1}-1\right)$ and obtain

$$
A_{t}(x) \leq c_{28} \mathbf{L}_{\alpha, p}^{2 t}[\omega](x) \quad \forall t \in(0,1) .
$$

Case 2: $t \geq 1$. We have

$$
\mathbf{L}_{\alpha, p}^{2 t}[\omega](x) \leq \int_{2 t}^{\infty}\left(\frac{\omega\left(B_{R}\right)}{s^{N-\alpha p}}\right)^{\frac{1}{p-1}} \frac{d s}{s}=c_{29} t^{-\frac{N-\alpha p}{p-1}},
$$

thus

$$
\begin{aligned}
A_{t}(x) & \leq c_{30} t^{\frac{\alpha p}{p-1}-1} t^{-\frac{(l \beta-p+1)(N-\alpha p)}{(p-1)^{2}}} \exp \left(\delta_{1} c_{31} t^{-\frac{\beta(N-\alpha p)}{p-1}}\right) \mathbf{L}_{\alpha, p}^{2 t}[\omega](x) \\
& \leq c_{32} t^{-1-\gamma} \mathbf{L}_{\alpha, p}^{2 t}[\omega](x)
\end{aligned}
$$

where $\gamma=\frac{1}{p-1}\left(\frac{l \beta(N-\alpha p)}{p-1}-N\right)>0$.

Therefore, $A_{t}(x) \leq c_{33}(t \vee 1)^{-1-\gamma} \mathbf{L}_{\alpha, p}^{2 t}[\omega](x)$ for all $t>0$. Therefore, from (2.17)

$$
\int_{0}^{+\infty}\left(\frac{\omega_{t}^{1}\left(B_{t}(x)\right)}{t^{N-\alpha p}}\right)^{\frac{1}{p-1}} \frac{d t}{t} \leq c_{34} \int_{0}^{\infty}(t \vee 1)^{-1-\gamma} \mathbf{L}_{\alpha, p}^{2 t}[\omega](x) d t .
$$

Using Fubini Theorem we get

$$
\begin{aligned}
\int_{0}^{+\infty}\left(\frac{\omega_{t}^{1}\left(B_{t}(x)\right)}{t^{N-\alpha p}}\right)^{\frac{1}{p-1}} \frac{d t}{t} & \leq c_{34} \int_{0}^{\infty} \int_{0}^{t / 2}(s \vee 1)^{-1-\gamma} d s\left(\frac{\omega\left(B_{t}(x)\right)}{t^{N-\alpha p}}\right)^{\frac{1}{p-1}} \frac{d t}{t} \\
& \leq c_{35} \int_{0}^{\infty}\left(\frac{\omega\left(B_{t}(x)\right)}{t^{N-\alpha p}}\right)^{\frac{1}{p-1}} \frac{d t}{t} \\
& =c_{35} \mathbf{W}_{\alpha, p}[\mu](x),
\end{aligned}
$$


which follows (2.15).

Step 2: Proof of (2.16). For $t>0, r \leq t$ and $y \in B_{t}(x)$ we have $B_{r}(y) \subset B_{2 t}(x)$, thus

$$
\omega_{t}^{2}\left(B_{t}(x)\right)=\int_{B_{t}(x)} H_{l}\left(\delta_{1} 2^{\beta}\left(\mathbf{W}_{\alpha, p}^{t}\left[\omega_{B_{2 t}(x)}\right](y)\right)^{\beta}\right) d y .
$$

By Theorem 2.2 there exists $c_{36}>0$ such that for $0<\delta_{1} \leq c_{36}, 0<t<2 R, z \in \mathbb{R}^{N}$,

$$
\int_{B_{4 t}(z)} \exp \left(\delta_{1} 2^{\beta}\left(\mathbf{W}_{\alpha, p}\left[\omega_{B_{2 t}(z)}\right](y)\right)^{\beta}\right) d y \leq c_{37} t^{N} .
$$

We take $0<\delta_{1} \leq c_{36}$.

Case 1: $x \in B_{R}$. If $0<t<2 R$, from (2.19) we get

$$
\omega_{t}^{2}\left(B_{t}(x)\right) \leq c_{37} t^{N} \leq c_{38} \omega\left(B_{4 t}(x)\right) .
$$

If $t \geq 2 R$, since for any $|y| \geq 2 R$,

$$
\mathbf{W}_{\alpha, p}[\omega](y)=\int_{|y| / 2}^{\infty}\left(\frac{\omega\left(B_{t}(y)\right)}{t^{N-\alpha p}}\right)^{\frac{1}{p-1}} \frac{d t}{t} \leq c_{39} \int_{|y| / 2}^{\infty} t^{-1-\frac{N-\alpha p}{p-1}} d t \leq c_{40}|y|^{-\frac{N-\alpha p}{p-1}},
$$

and thanks to (2.19) we have

$$
\begin{aligned}
\omega_{t}^{2}\left(B_{t}(x)\right) & \leq \int_{B_{2 R}} \exp \left(\delta_{1} 2^{\beta}\left(\mathbf{W}_{\alpha, p}\left[\omega_{B_{R}}\right](y)\right)^{\beta}\right) d y+\int_{\mathbb{R}^{N} \backslash B_{2 R}} H_{l}\left(\delta_{1} 2^{\beta}\left(\mathbf{W}_{\alpha, p}[\omega](y)\right)^{\beta}\right) d y \\
& \leq c_{41} R^{N}+\int_{\mathbb{R}^{N} \backslash B_{2 R}} H_{l}\left(c_{42}|y|^{-\frac{\beta(N-\alpha p)}{p-1}}\right) d y \\
& \leq c_{43}+c_{43} \int_{\mathbb{R}^{N} \backslash B_{2 R}}|y|^{-\frac{l \beta(N-\alpha p)}{p-1}} d y=c_{43}+c_{44} R^{N-\frac{l \beta(N-\alpha p)}{p-1}} \\
& \leq c_{45}\left|B_{4 t}(x) \cap B_{R}\right| \leq c_{46} \omega\left(B_{4 t}(x)\right) .
\end{aligned}
$$

From this we also have $H_{l}\left(\delta_{1}\left(\mathbf{W}_{\alpha, p}[\omega]\right)^{\beta}\right) \in L^{1}\left(\mathbb{R}^{N}\right)$.

Case 2: $x \in \mathbb{R}^{N} \backslash B_{R}$. If $|x|>R+t$ then $\omega_{t}^{2}\left(B_{t}(x)\right)=0$. Next we consider the case $R<|x| \leq$ $R+t$. If $0<t<2 R$, we have $B_{t / 2}\left(\left(R-\frac{t}{2}\right) \frac{x}{|x|}\right) \subset B_{4 t}(x) \cap B_{R}$; thus from (2.19) we get

$$
\omega_{t}^{2}\left(B_{t}(x)\right) \leq c_{47} t^{N}=c_{48}\left|B_{t / 2}\left(\left(R-\frac{t}{2}\right) \frac{x}{|x|}\right)\right| \leq c_{48}\left|B_{4 t}(x) \cap B_{R}\right| \leq c_{49} \omega\left(B_{4 t}(x)\right) .
$$

If $t \geq 2 R$, as in Case 1 we also obtain $\omega_{t}^{2}\left(B_{t}(x)\right) \leq c_{50} \omega\left(B_{4 t}(x)\right)$ since $B_{R} \subset B_{4 t}(x)$. Hence, we get (2.16). Therefore, the result follows with $\delta_{1}=\left(\frac{1}{2 c_{26}}\left(\frac{\alpha p}{p-1}-1\right)\right) \wedge c_{36}$.

In the next result we obtain estimate on a sequence of solutions of Wolff integral inequations obtained by induction. 
Theorem 2.5 Assume that the assumptions on $\alpha, p, l, a, \beta, \varepsilon, f, \mu_{1}$ and $\mu$ of Theorem 1.5 are fulfilled and $R, K$ are positive real numbers. Suppose that $\left\{u_{m}\right\}$ is a sequence of nonnegative measurable functions in $\mathbb{R}^{N}$ that satisfies

$$
\begin{aligned}
u_{m+1} & \leq K \mathbf{W}_{\alpha, p}^{R}\left[P_{l, a, \beta}\left(u_{m}\right)+\mu\right]+f \quad \forall m \in \mathbb{N}, \\
u_{0} & \leq K \mathbf{W}_{\alpha, p}^{R}[\mu]+f .
\end{aligned}
$$

Then there exists $M>0$ depending on $N, \alpha, p, l, a, \beta, \varepsilon, K$ and $R$ such that if

$$
\left\|\mathbf{M}_{\alpha p, R}^{\frac{(p-1)(\beta-1)}{\beta}}[\mu]\right\|_{L^{\infty}\left(\mathbb{R}^{N}\right)} \leq M \quad \text { and } \quad\left\|\mathbf{M}_{\alpha p, R}^{\frac{(p-1)(\beta-1)}{\beta}}\left[\mu_{1}\right]\right\|_{L^{\infty}\left(\mathbb{R}^{N}\right)} \leq M,
$$

there holds

$$
P_{l, a, \beta}\left(4 c_{p} K \mathbf{W}_{\alpha, p}^{R}\left[\omega_{1}\right]+4 c_{p} K \mathbf{W}_{\alpha, p}^{R}\left[\omega_{2}\right]+f\right) \in L_{l o c}^{1}\left(\mathbb{R}^{N}\right),
$$

and

$$
u_{m} \leq 2 c_{p} K \mathbf{W}_{\alpha, p}^{R}\left[\omega_{1}\right]+2 c_{p} K \mathbf{W}_{\alpha, p}^{R}\left[\omega_{2}\right]+f \quad \forall m \in \mathbb{N},
$$

where

$$
\begin{aligned}
& \omega_{1}=M\left\|\mathbf{M}_{\alpha p, R}^{\frac{(p-1)(\beta-1)}{\beta}}[1]\right\|_{L^{\infty}\left(\mathbb{R}^{N}\right)}^{-1}+\mu, \\
& \omega_{2}=M\left\|\mathbf{M}_{\alpha p, R}^{\frac{(p-1)(\beta-1)}{\beta}}[1]\right\|_{L^{\infty}\left(\mathbb{R}^{N}\right)}^{-1}+\mu_{1},
\end{aligned}
$$

and $c_{p}=1 \vee 4^{\frac{2-p}{p-1}}$.

Furthermore, if $f \equiv 0$ then (2.21) and (2.22) are satisfied with $\omega_{2} \equiv 0$.

Proof. The proof is based upon Theorems 2.3 and 2.4. Set $c_{a, \varepsilon}=2\left(1-\left(\frac{a}{a+\varepsilon}\right)^{1 / \beta}\right)^{-1}$ and $\bar{a}=a\left(4 c_{a, \varepsilon} c_{p} K\right)^{\beta}$. If $0<M \leq 1$ we define $\omega_{1}$ and $\omega_{2}$ by (2.23) and (2.24) respectively. We now assume

$$
\left\|\mathbf{M}_{\alpha p, R}^{\frac{(p-1)(\beta-1)}{\beta}}[\mu]\right\|_{L^{\infty}\left(\mathbb{R}^{N}\right)} \leq M \quad \text { and }\left\|\mathbf{M}_{\alpha p, R}^{\frac{(p-1)(\beta-1)}{\beta}}\left[\mu_{1}\right]\right\|_{L^{\infty}\left(\mathbb{R}^{N}\right)} \leq M .
$$

We prove first that

$$
\mathbf{W}_{\alpha, p}^{R}\left[H_{l}\left(\bar{a}\left(\mathbf{W}_{\alpha, p}^{R}\left[\omega_{i}\right]\right)^{\beta}\right)\right] \leq \mathbf{W}_{\alpha, p}^{R}\left[\omega_{i}\right] \quad \text { for } i=1,2 .
$$

By Theorem 2.3 , there exist $c, \delta_{0}>0$ independent on $\mu$ such that $\exp \left(\delta_{0}\left(\mathbf{W}_{\alpha, p}^{R}\left[M^{-1} \omega_{i}\right]\right)^{\beta}\right)$ is locally integrable in $\mathbb{R}^{N}$ and

$$
\mathbf{W}_{\alpha, p}^{R}\left[\exp \left(\delta_{0}\left(\mathbf{W}_{\alpha, p}^{R}\left[M^{-1} \omega_{i}\right]\right)^{\beta}\right)\right] \leq c \mathbf{W}_{\alpha, p}^{R}\left[M^{-1} \omega_{i}\right] \quad \text { in } \mathbb{R}^{N} .
$$

Since $\theta^{-l} H_{l}(s) \leq H_{l}\left(\theta^{-1} s\right)$ for all $s \geq 0$ and $0<\theta \leq 1$, it follows

$$
\begin{aligned}
\mathbf{W}_{\alpha, p}^{R}\left[M^{-\frac{1}{2}\left(\frac{\beta l}{p-1}+1\right)} H_{l}\left(\delta_{0} M^{-\frac{1}{2}\left(\frac{\beta}{p-1}-\frac{1}{l}\right)}\left(\mathbf{W}_{\alpha, p}^{R}\left[\omega_{i}\right]\right)^{\beta}\right)\right] & \leq \mathbf{W}_{\alpha, p}^{R}\left[H_{l}\left(\delta_{0} M^{-\frac{\beta}{p-1}}\left(\mathbf{W}_{\alpha, p}^{R}\left[\omega_{i}\right]\right)^{\beta}\right)\right] \\
& \leq \mathbf{W}_{\alpha, p}^{R}\left[\exp \left(\delta_{0}\left(\mathbf{W}_{\alpha, p}^{R}\left[M^{-1} \omega_{i}\right]\right)^{\beta}\right)\right] \\
& \leq c M^{-\frac{1}{p-1}} \mathbf{W}_{\alpha, p}^{R}\left[\omega_{i}\right] .
\end{aligned}
$$


Hence,

$$
\mathbf{W}_{\alpha, p}^{R}\left[H_{l}\left(\delta_{0} M^{-\frac{1}{2}\left(\frac{\beta}{p-1}-\frac{1}{l}\right)}\left(\mathbf{W}_{\alpha, p}^{R}\left[\omega_{i}\right]\right)^{\beta}\right)\right] \leq c M^{\frac{1}{2(p-1)}\left(\frac{\beta l}{p-1}-1\right)} \mathbf{W}_{\alpha, p}^{R}\left[\omega_{i}\right] .
$$

Therefore (2.25) is achieved if we prove

$$
\bar{a} \leq \delta_{0} M^{-\frac{1}{2}\left(\frac{\beta}{p-1}-\frac{1}{l}\right)} \text { and } c M^{\frac{1}{2(p-1)}\left(\frac{\beta l}{p-1}-1\right)} \leq 1,
$$

which is equivalent to

$$
M \leq\left(\delta_{0} \bar{a}^{-1}\right)^{\left(\frac{1}{2}\left(\frac{\beta}{p-1}-\frac{1}{l}\right)\right)^{-1}} \wedge c^{-\left(\frac{1}{2(p-1)}\left(\frac{\beta l}{p-1}-1\right)\right)^{-1}} .
$$

Thus, we choose $M=1 \wedge\left(\delta_{0} \bar{a}^{-1}\right)^{\left(\frac{1}{2}\left(\frac{\beta}{p-1}-\frac{1}{l}\right)\right)^{-1}} \wedge c^{-\left(\frac{1}{2(p-1)}\left(\frac{\beta l}{p-1}-1\right)\right)^{-1}}$; we obtain (2.25) and the fact that $H_{l}\left(\bar{a}\left(\mathbf{W}_{\alpha, p}^{R}\left[\omega_{i}\right]\right)^{\beta}\right) \in L_{l o c}^{1}\left(\mathbb{R}^{N}\right)$.

Now, we prove (2.22) by induction. Clearly, (2.22) holds with $m=0$. Next we assume that (2.22) holds with $m=n$, and we claim that

$$
u_{n+1} \leq 2 c_{p} K \mathbf{W}_{\alpha, p}^{R}\left[\omega_{1}\right]+2 c_{p} K \mathbf{W}_{\alpha, p}^{R}\left[\omega_{2}\right]+f .
$$

In fact, since (2.22) holds with $m=n$ and $P_{l, a, \beta}$ is convex, we have

$$
\begin{aligned}
P_{l, a, \beta}\left(u_{n}\right) & \leq P_{l, a, \beta}\left(4 c_{p} K \mathbf{W}_{\alpha, p}^{R}\left[\omega_{1}\right]+4 c_{p} K \mathbf{W}_{\alpha, p}^{R}\left[\omega_{2}\right]+f\right) \\
& \leq P_{l, a, \beta}\left(4 c_{a, \varepsilon} c_{p} K \mathbf{W}_{\alpha, p}^{R}\left[\omega_{1}\right]\right)+P_{l, \varepsilon, a}\left(4 c_{a, \varepsilon} c_{p} K \mathbf{W}_{\alpha, p}^{R}\left[\omega_{2}\right]\right)+P_{l, a, \beta}\left(\left(1+\frac{\varepsilon}{a}\right)^{1 / \beta} f\right) \\
& =H_{l}\left(\bar{a}\left(\mathbf{W}_{\alpha, p}^{R}\left[\omega_{1}\right]\right)^{\beta}\right)+H_{l}\left(\bar{a}\left(\mathbf{W}_{\alpha, p}^{R}\left[\omega_{2}\right]\right)^{\beta}\right)+P_{l, a+\varepsilon, \beta}(f) .
\end{aligned}
$$

From this we derive (2.21). By the definition of $u_{n+1}$ and the sub-additive property of $\mathbf{W}_{\alpha, p}^{R}[\cdot]$, we obtain

$$
\begin{gathered}
u_{n+1} \leq K \mathbf{W}_{\alpha, p}^{R}\left[H_{l}\left(\bar{a}\left(\mathbf{W}_{\alpha, p}^{R}\left[\omega_{1}\right]\right)^{\beta}\right)+H_{l}\left(\bar{a}\left(\mathbf{W}_{\alpha, p}^{R}\left[\omega_{2}\right]\right)^{\beta}\right)+P_{l, a+\varepsilon, \beta}(f)+\mu\right]+f \\
\leq c_{p} K \mathbf{W}_{\alpha, p}^{R}\left[H_{l}\left(\bar{a}\left(\mathbf{W}_{\alpha, p}^{R}\left[\omega_{1}\right]\right)^{\beta}\right)\right]+c_{p} K \mathbf{W}_{\alpha, p}^{R}\left[H_{l}\left(\bar{a}\left(\mathbf{W}_{\alpha, p}^{R}\left[\omega_{2}\right]\right)^{\beta}\right)\right] \\
+c_{p} K \mathbf{W}_{\alpha, p}^{R}\left[P_{l, a+\varepsilon, \beta}(f)\right]+c_{p} K \mathbf{W}_{\alpha, p}^{R}[\mu]+f .
\end{gathered}
$$

Hence follows (2.26) from (2.25). This completes the proof of the theorem.

The next result is obtained by an easy adaptation of the proof Theorem 2.5 .

Theorem 2.6 Assume that the assumptions on $\alpha, p, a, l, \beta, \varepsilon, f, \mu_{1}$ and $\mu$ of Theorem 1.6 are fulfilled and $R, K$ are positive real numbers. Suppose that $\left\{u_{m}\right\}$ is a sequence of nonnegative measurable functions in $\mathbb{R}^{N}$ that satisfies

$$
\begin{aligned}
u_{m+1} & \leq K \mathbf{W}_{\alpha, p}\left[P_{l, a, \beta}\left(u_{m}\right)+\mu\right]+f \quad \forall m \in \mathbb{N}, \\
u_{0} & \leq K \mathbf{W}_{\alpha, p}[\mu]+f .
\end{aligned}
$$


Then there exists $M>0$ depending on $N, \alpha, p, l, a, \beta, \varepsilon, K$ and $R$ such that if

$$
\left\|\mathbf{M}_{\alpha p}^{\frac{(p-1)(\beta-1)}{\beta}}[\mu]\right\|_{L^{\infty}\left(\mathbb{R}^{N}\right)} \leq M \quad \text { and }\left\|\mathbf{M}_{\alpha p}^{\frac{(p-1)(\beta-1)}{\beta}}\left[\mu_{1}\right]\right\|_{L^{\infty}\left(\mathbb{R}^{N}\right)} \leq M,
$$

there holds

$$
P_{l, a, \beta}\left(4 c_{p} K \mathbf{W}_{\alpha, p}\left[\omega_{3}\right]+4 c_{p} K \mathbf{W}_{\alpha, p}\left[\omega_{4}\right]+f\right) \in L^{1}\left(\mathbb{R}^{N}\right),
$$

and

$$
u_{m} \leq 2 c_{p} K \mathbf{W}_{\alpha, p}\left[\omega_{3}\right]+2 c_{p} K \mathbf{W}_{\alpha, p}\left[\omega_{4}\right]+f \quad \forall m \in \mathbb{N},
$$

where

$$
\omega_{3}=M\left\|\mathbf{M}_{\alpha p}^{\frac{(p-1)(\beta-1)}{\beta}}\left[\chi_{B_{R}}\right]\right\|_{L^{\infty}\left(\mathbb{R}^{N}\right)}^{-1} \chi_{B_{R}}+\mu
$$

and

$$
\omega_{4}=M\left\|\mathbf{M}_{\alpha p}^{\frac{(p-1)(\beta-1)}{\beta}}\left[\chi_{B_{R}}\right]\right\|_{L^{\infty}\left(\mathbb{R}^{N}\right)}^{-1} \chi_{B_{R}}+\mu_{1} .
$$

Furthermore, if $f \equiv 0$ then (2.28) and (2.29) are satisfied with $\omega_{4} \equiv 0$.

Let $P \in C\left(\mathbb{R}^{+}\right)$be a decreasing positive function. The $(\alpha, P)$-Orlicz-Bessel capacity of a Borel set $E \subset \mathbb{R}^{N}$ is defined by (see [1, Sect 2.6])

$$
\operatorname{Cap}_{\mathbf{G}_{\alpha}, P}(E)=\inf \left\{\int_{\mathbb{R}^{N}} P(f): \mathbf{G}_{\alpha} * f \geq \chi_{E}, f \geq 0, P(f) \in L^{1}\left(\mathbb{R}^{N}\right)\right\},
$$

and the $(\alpha, P)$-Orlicz-Riesz capacity

$$
\operatorname{Cap}_{\mathbf{I}_{\alpha}, P}(E)=\inf \left\{\int_{\mathbb{R}^{N}} P(f): \mathbf{I}_{\alpha} * f \geq \chi_{E}, f \geq 0, P(f) \in L^{1}\left(\mathbb{R}^{N}\right)\right\} .
$$

Theorem 2.7 Let $\alpha>0, p>1, a>0, c>0, l \in \mathbb{N}^{*}$ and $\beta \geq 1$ such that $l \beta>p-1$ and $0<\alpha p<N$. Let $\mu \in \mathfrak{M}^{+}\left(\mathbb{R}^{N}\right)$.

1. Let $0<R \leq \infty$. If $u$ is a nonnegative Borel function in $\mathbb{R}^{N}$ such that $P_{l, a, \beta}(u)$ is locally integrable in $\mathbb{R}^{N}$ and

$$
u(x) \geq c \mathbf{W}_{\alpha, p}^{R}\left[P_{l, a, \beta}(u)+\mu\right](x) \quad \forall x \in \mathbb{R}^{N},
$$

then the following statements holds.

(i) If $R<\infty$, there exists a positive constant $C_{1}$ depending on $N, \alpha, p, l, a, \beta, c$ and $R$ such that

$$
\int_{E} P_{l, a, \beta}(u) d x+\mu(E) \leq C_{1} \operatorname{Cap}_{\mathbf{G}_{\alpha p}, Q_{p}^{*}}(E) \quad \forall E \subset \mathbb{R}^{N}, E \text { Borel. }
$$

(ii) If $R=\infty$, there exists a positive constant $C_{2}$ depending on $N, \alpha, p, l, a, \beta, c$ such that

$$
\int_{E} P_{l, a, \beta}(u) d x+\mu(E) \leq C_{2} \operatorname{Cap}_{\mathbf{I}_{\alpha p}, Q_{p}^{*}}(E) \quad \forall E \subset \mathbb{R}^{N}, E \text { Borel. }
$$


2. Let $\Omega$ be a bounded domain in $\mathbb{R}^{N}, \mu \in \mathfrak{M}^{+}(\Omega)$ and $\delta \in(0,1)$. If $u$ is a nonnegative Borel function in $\Omega$ such that $P_{l, a, \beta}(u)$ is locally integrable in $\Omega$ and

$$
u(x) \geq c \mathbf{W}_{\alpha, p}^{\delta d(x, \partial \Omega)}\left[P_{l, a, \beta}(u)+\mu\right](x) \quad \forall x \in \Omega,
$$

then, for any compact set $K \subset \Omega$, there exists a positive constant $C_{3}$ depending on $N, \alpha, p, l, a, \beta, c, \delta$ and $\operatorname{dist}(K, \partial \Omega)$ such that

$$
\int_{E} P_{l, a, \beta}(u) d x+\mu(E) \leq C_{3} \operatorname{Cap}_{\mathbf{G}_{\alpha_{p}, Q_{p}^{*}}}(E) \quad \forall E \subset K, E \text { Borel, }
$$

where $Q_{p}^{*}$ is the complementary function to $Q_{p}$.

Proof. Set $d \omega=P_{l, a, \beta}(u) d x+d \mu$.

1. We have

$$
P_{l, a, \beta}\left(c \mathbf{W}_{\alpha, p}^{R}[\omega]\right) d x \leq d \omega \text { in } \mathbb{R}^{N} .
$$

Let $M_{\omega}$ denote the centered Hardy-Littlewood maximal function which is defined for any $f \in$ $L_{l o c}^{1}\left(\mathbb{R}^{N}, d \omega\right)$ by

$$
M_{\omega} f(x)=\sup _{t>0} \frac{1}{\omega\left(B_{t}(x)\right)} \int_{B_{t}(x)}|f| d \omega .
$$

If $E \subset \mathbb{R}^{N}$ is a Borel set, we have

$$
\int_{\mathbb{R}^{N}}\left(M_{\omega} \chi_{E}\right)^{\frac{l \beta}{p-1}} P_{l, a, \beta}\left(c \mathbf{W}_{\alpha, p}^{R}[\omega]\right) d x \leq \int_{\mathbb{R}^{N}}\left(M_{\omega} \chi_{E}\right)^{\frac{l \beta}{p-1}} d \omega .
$$

Since $M_{\omega}$ is bounded on $L^{s}\left(\mathbb{R}^{N}, d \omega\right), s>1$, we deduce from Fefferman's result [1] that

$$
\int_{\mathbb{R}^{N}}\left(M_{\omega} \chi_{E}\right)^{\frac{l \beta}{p-1}} P_{l, a, \beta}\left(c \mathbf{W}_{\alpha, p}^{R}[\omega]\right) d x \leq c_{51} \omega(E),
$$

for some constant $c_{51}$ only depends on $N$ and $\frac{l \beta}{p-1}$. Since $M_{\omega} \chi_{E} \leq 1$, we derive

$$
\begin{aligned}
\left(M_{\omega} \chi_{E}(x)\right)^{\frac{l \beta}{p-1}} P_{l, a, \beta}\left(c \mathbf{W}_{\alpha, p}^{R}[\omega](x)\right) & \geq P_{l, a, \beta}\left(c\left(M_{\omega} \chi_{E}(x)\right)^{\frac{1}{p-1}} \mathbf{W}_{\alpha, p}^{R}[\omega](x)\right) \\
& \geq P_{l, a, \beta}\left(c \mathbf{W}_{\alpha, p}^{R}\left[\omega_{E}\right](x)\right),
\end{aligned}
$$

where $\omega_{E}=\chi_{E} \omega$. Thus

$$
\int_{\mathbb{R}^{N}} P_{l, a, \beta}\left(c \mathbf{W}_{\alpha, p}^{R}\left[\omega_{E}\right]\right) d x \leq c_{51} \omega(E) \quad \forall E \subset \mathbb{R}^{N}, E \text { Borel. }
$$

From (2.1), (2.2) and (2.3) we get

$$
\int_{\mathbb{R}^{N}} P_{l, a, \beta}\left(c \mathbf{W}_{\alpha, p}^{R}\left[\omega_{E}\right](x)\right) d x \geq \int_{\mathbb{R}^{N}} Q_{p}\left(c_{52} \mathbf{G}_{\alpha p}\left[\omega_{E}\right](x)\right) d x \quad \text { if } R<\infty,
$$

and

$$
\int_{\mathbb{R}^{N}} P_{l, a, \beta}\left(c \mathbf{W}_{\alpha, p}^{R}\left[\omega_{E}\right](x)\right) d x \geq \int_{\mathbb{R}^{N}} Q_{p}\left(c_{53} \mathbf{I}_{\alpha p}\left[\omega_{E}\right](x)\right) d x \quad \text { if } R=\infty
$$


where $Q_{p}$ is defined by (1.13) and $c_{52}=\left(c_{2} \beta\right)^{-1} a^{\frac{p-1}{\beta}} c^{p-1}$ if $p \neq 2, c_{52}=c_{3}^{-1} a^{\frac{1}{\beta}} c$ if $p=2$ (the constants $c_{2}, c_{3}$ defined in (2.2) and (2.3), depend on $R$, therefore $c_{52}=c_{52}\left(r_{K}\right)$ ) and $c_{53}=\left(c_{1} \beta\right)^{-1} a^{\frac{p-1}{\beta}} c^{p-1}$ if $p \neq 2, c_{53}=a^{\frac{1}{\beta}} c$ if $p=2$. Thus, from (2.37) we obtain that for all Borel set $E \subset \mathbb{R}^{N}$ there holds

$$
\int_{\mathbb{R}^{N}} Q_{p}\left(c_{52} \mathbf{G}_{\alpha p}\left[\omega_{E}\right](x)\right) d x \leq c_{51} \omega(E) \text { if } R<\infty
$$

and

$$
\int_{\mathbb{R}^{N}} Q_{p}\left(c_{53} \mathbf{I}_{\alpha p}\left[\omega_{E}\right](x)\right) d x \leq c_{51} \omega(E) \text { if } R=\infty .
$$

We recall that $Q_{p}^{*}(s)=\sup _{t>0}\left\{s t-Q_{p}(t)\right\}$ satisfies the sub-additivity $\Delta_{2}$-condition (see Chapter 2 in [19]).

(i) We assume $R<\infty$. For every $f \geq 0, Q_{p}^{*}(f) \in L^{1}(\Omega)$ such that $\mathbf{G}_{\alpha p} * f \geq \chi_{E}$, we have

$$
\begin{aligned}
\omega(E) & \leq \int_{\mathbb{R}^{N}} \mathbf{G}_{\alpha p} * f d \omega_{E}=\left(2 c_{51}\right)^{-1} \int_{\mathbb{R}^{N}}\left(c_{52} \mathbf{G}_{\alpha p}\left[\omega_{E}\right]\right)\left(2 c_{51} c_{52}^{-1} f\right) d x \\
& \leq\left(2 c_{51}\right)^{-1} \int_{\mathbb{R}^{N}} Q_{p}\left(c_{52} \mathbf{G}_{\alpha p}\left[\omega_{E}\right]\right) d x+\left(2 c_{51}\right)^{-1} \int_{\mathbb{R}^{N}} Q_{p}^{*}\left(2 c_{51} c_{52}^{-1} f\right) d x \\
& \leq 2^{-1} \omega(E)+c_{54} \int_{\mathbb{R}^{N}} Q_{p}^{*}(f) d x,
\end{aligned}
$$

the last inequality following from the $\Delta_{2}$-condition. Notice that $c_{54}$, as well as the next constant $c_{55}$, depends on $r_{K}$. Thus,

$$
\omega(E) \leq 2 c_{54} \int_{\mathbb{R}^{N}} Q_{p}^{*}(f) d x .
$$

Then, we get

$$
\omega(E) \leq c_{55} \operatorname{Cap}_{\mathbf{G}_{\alpha p}, Q_{p}^{*}}(E) \quad \forall E \subset \mathbb{R}^{N}, E \text { Borel. }
$$

Which implies (2.33).

(ii) We assume $R=\infty$. For every $f \geq 0, Q_{p}^{*}(f) \in L^{1}(\Omega)$ such that $\mathbf{I}_{\alpha p} * f \geq \chi_{E}$, since $\mathbf{I}_{\alpha p} * \omega_{E}=\mathbf{I}_{\alpha p}\left[\omega_{E}\right]$, as above we have

$$
\begin{aligned}
\omega(E) & \leq \int_{\mathbb{R}^{N}} \mathbf{I}_{\alpha p} * f d \omega_{E}=\int_{\mathbb{R}^{N}}\left(\mathbf{I}_{\alpha p} * \omega_{E}\right) f d x=\int_{\mathbb{R}^{N}} \mathbf{I}_{\alpha p}\left[\omega_{E}\right] f d x \\
& \leq 2^{-1} \omega(E)+c_{56} \int_{\mathbb{R}^{N}} Q_{p}^{*}(f) d x,
\end{aligned}
$$

Then, it follows (2.34).

2. Let $K \subset \Omega$ be compact. Set $r_{K}=\operatorname{dist}(K, \partial \Omega)$ and $\Omega_{K}=\left\{x \in \Omega: d(x, K)<r_{K} / 2\right\}$. We have

$$
P_{l, a, \beta}\left(c \mathbf{W}_{\alpha, p}^{\delta d(x, \partial \Omega)}[\omega]\right) d x \leq d \omega \text { in } \Omega .
$$

Thus, for any Borel set $E \subset K$

$$
\int_{\Omega}\left(M_{\omega} \chi_{E}\right)^{\frac{l \beta}{p-1}} P_{l, a, \beta}\left(c \mathbf{W}_{\alpha, p}^{\delta d(x, \partial \Omega)}[\omega]\right) d x \leq \int_{\Omega}\left(M_{\omega} \chi_{E}\right)^{\frac{l \beta}{p-1}} d \omega .
$$


As above we get

$$
\int_{\Omega} P_{l, a, \beta}\left(c \mathbf{W}_{\alpha, p}^{\delta d(x, \partial \Omega)}\left[\omega_{E}\right](x)\right) d x \leq c_{51} \omega(E) \quad \forall E \subset K, E \text { Borel. }
$$

Note that if $x \in \Omega$ and $d(x, \partial \Omega) \leq r_{K} / 8$, then $B_{t}(x) \subset \Omega \backslash \Omega_{K}$ for all $t \in(0, \delta d(x, \partial \Omega))$; indeed, for all $y \in B_{t}(x)$

$$
d(y, \partial \Omega) \leq d(x, \partial \Omega)+|x-y|<(1+\delta) d(x, \partial \Omega)<\frac{1}{4} r_{K},
$$

thus

$$
d(y, K) \geq d(K, \partial \Omega)-d(y, \partial \Omega)>\frac{3}{4} r_{K}>\frac{1}{2} r_{K},
$$

which implies $y \notin \Omega_{K}$. We deduce that

$$
\mathbf{W}_{\alpha, p}^{\delta d(x, \partial \Omega)}\left[\omega_{E}\right](x) \geq \mathbf{W}_{\alpha, p}^{\frac{\delta}{8} r_{K}}\left[\omega_{E}\right](x) \quad \forall x \in \Omega,
$$

and

$$
\mathbf{W}_{\alpha, p}^{\frac{\delta}{8} r_{K}}\left[\omega_{E}\right](x)=0 \quad \forall x \in \Omega^{c} .
$$

Hence we obtain from (2.38),

$$
\int_{\mathbb{R}^{N}} P_{l, a, \beta}\left(c \mathbf{W}_{\alpha, p}^{\frac{\delta}{8} r_{K}}\left[\omega_{E}\right](x)\right) d x \leq c_{51} \omega(E) \quad \forall E \subset K, E \text { Borel. }
$$

As above we also obtain

$$
\omega(E) \leq c_{57} \operatorname{Cap}_{\mathbf{G}_{\alpha p}, Q_{p}^{*}}(E) \quad \forall E \subset K, E \text { Borel, }
$$

where the positive constant $c_{57}$ depends on $r_{K}$. Inequality (2.36) follows and this completes the proof of the Theorem.

Proof of Theorem 1.5. Consider the sequence $\left\{u_{m}\right\}_{m \geq 0}$ of nonnegative functions defined by $u_{0}=f$ and

$$
u_{m+1}=\mathbf{W}_{\alpha, p}^{R}\left[P_{l, a, \beta}\left(u_{m}\right)\right]+f \quad \text { in } \quad \mathbb{R}^{N} \quad \forall m \geq 0 .
$$

By Theorem 2.5, there exists $M>0$ depending on $N, \alpha, p, l, a, \beta, \varepsilon$ and $R$ such that if (1.28) holds, then $\left\{u_{m}\right\}_{m \geq 0}$ is well defined and (2.21) and (2.22) are satisfied. It is easy to see that $\left\{u_{m}\right\}$ is nondecreasing. Hence, thanks to the dominated convergence theorem, we obtain that $u(x)=\lim _{m \rightarrow \infty} u_{m}(x)$ is a solution of equation (1.29) which satisfies (1.30).

Conversely, we obtain (1.31) directly from Theorem 2.7, Part 1, (i).

Proof of Theorem 1.6. The proof is similar to the previous one by using Theorem 2.6 and Theorem 2.7 Part 1, (ii). 


\section{Quasilinear Dirichlet problems}

Let $\Omega$ be a bounded domain in $\mathbb{R}^{N}$. If $\mu \in \mathfrak{M}_{b}(\Omega)$, we denote by $\mu^{+}$and $\mu^{-}$respectively its positive and negative parts in the Jordan decomposition. We denote by $\mathfrak{M}_{0}(\Omega)$ the space of measures in $\Omega$ which are absolutely continuous with respect to the $c_{1, p}^{\Omega}$-capacity defined on a compact set $K \subset \Omega$ by

$$
c_{1, p}^{\Omega}(K)=\inf \left\{\int_{\Omega}|\nabla \varphi|^{p} d x: \varphi \geq \chi_{K}, \varphi \in C_{c}^{\infty}(\Omega)\right\} .
$$

We also denote $\mathfrak{M}_{s}(\Omega)$ the space of measures in $\Omega$ with support on a set of zero $c_{1, p}^{\Omega}$-capacity. Classically, any $\mu \in \mathfrak{M}_{b}(\Omega)$ can be written in a unique way under the form $\mu=\mu_{0}+\mu_{s}$ where $\mu_{0} \in \mathfrak{M}_{0}(\Omega) \cap \mathfrak{M}_{b}(\Omega)$ and $\mu_{s} \in \mathfrak{M}_{s}(\Omega)$. It is well known that any $\mu_{0} \in \mathfrak{M}_{0}(\Omega) \cap \mathfrak{M}_{b}(\Omega)$ can be written under the form $\mu_{0}=f-\operatorname{div} g$ where $f \in L^{1}(\Omega)$ and $g \in L^{p^{\prime}}\left(\Omega, \mathbb{R}^{N}\right)$.

For $k>0$ and $s \in \mathbb{R}$ we set $T_{k}(s)=\max \{\min \{s, k\},-k\}$. If $u$ is a measurable function defined in $\Omega$, finite a.e. and such that $T_{k}(u) \in W_{l o c}^{1, p}(\Omega)$ for any $k>0$, there exists a measurable function $v: \Omega \rightarrow \mathbb{R}^{N}$ such that $\nabla T_{k}(u)=\chi_{|u| \leq k} v$ a.e. in $\Omega$ and for all $k>0$. We define the gradient $\nabla u$ of $u$ by $v=\nabla u$. We recall the definition of a renormalized solution given in [10].

Definition 3.1 Let $\mu=\mu_{0}+\mu_{s} \in \mathfrak{M}_{b}(\Omega)$. A measurable function $u$ defined in $\Omega$ and finite a.e. is called a renormalized solution of

$$
\begin{aligned}
-\Delta_{p} u & =\mu & & \text { in } \Omega, \\
u & =0 & & \text { on } \partial \Omega,
\end{aligned}
$$

if $T_{k}(u) \in W_{0}^{1, p}(\Omega)$ for any $k>0,|\nabla u|^{p-1} \in L^{r}(\Omega)$ for any $0<r<\frac{N}{N-1}$, and $u$ has the property that for any $k>0$ there exist $\lambda_{k}^{+}$and $\lambda_{k}^{-}$belonging to $\mathfrak{M}_{b}^{+} \cap \mathfrak{M}_{0}(\Omega)$, respectively concentrated on the sets $u=k$ and $u=-k$, with the property that $\mu_{k}^{+} \rightarrow \mu_{s}^{+}, \mu_{k}^{-} \rightarrow \lambda_{s}^{-}$in the narrow topology of measures and such that

$$
\int_{\{|u|<k\}}|\nabla u|^{p-2} \nabla u . \nabla \varphi d x=\int_{\{|u|<k\}} \varphi d \mu_{0}+\int_{\Omega} \varphi d \lambda_{k}^{+}-\int_{\Omega} \varphi d \lambda_{k}^{-},
$$

for every $\varphi \in W_{0}^{1, p}(\Omega) \cap L^{\infty}(\Omega)$.

Remark 3.2 We recall that if $u$ is a renormalized solution to problem (3.1), then $\frac{|\nabla u|^{p}}{(|u|+1)^{r}} \in$ $L^{1}(\Omega)$ for all $r>1$. From this it follows by Hölder's inequality that $u \in W_{0}^{1, p_{1}}(\Omega)$ for all $1 \leq p_{1}<p$ provided $e^{a|u|} \in L^{1}(\Omega)$ for some $a>0$. Furthermore, $u \geq 0$ a.e. in $\Omega$ if $\mu \in \mathfrak{M}_{b}^{+}(\Omega)$.

The following general stability result has been proved in [10, Th 4.1].

Theorem 3.3 Let $\mu=\mu_{0}+\mu_{s}^{+}-\mu_{s}^{-}$, with $\mu_{0}=F-\operatorname{div} g \in \mathfrak{M}_{0}(\Omega)$ and $\mu_{s}^{+}$, $\mu_{s}^{-}$belonging to $\mathfrak{M}_{s}^{+}(\Omega)$. Let $\mu_{n}=F_{n}-\operatorname{div} g_{n}+\rho_{n}-\eta_{n}$ with $F_{n} \in L^{1}(\Omega), g_{n} \in\left(L^{p^{\prime}}(\Omega)\right)^{N}$ and $\rho_{n}$, $\eta_{n}$ belonging to $\mathfrak{M}_{b}^{+}(\Omega)$. Assume that $\left\{F_{n}\right\}$ converges to $F$ weakly in $L^{1}(\Omega),\left\{g_{n}\right\}$ converges to $g$ strongly in $\left(L^{p^{\prime}}(\Omega)\right)^{N}$ and $\left(\operatorname{div} g_{n}\right)$ is bounded in $\mathfrak{M}_{b}(\Omega)$; assume also that $\left\{\rho_{n}\right\}$ converges to $\mu_{s}^{+}$and $\left\{\eta_{n}\right\}$ to $\mu_{s}^{-}$in the narrow topology. If $\left\{u_{n}\right\}$ is a sequence of renormalized solutions of (3.1) with data $\mu_{n}$, then, up to a subsequence, it converges a.e. in $\Omega$ to a renormalized solution $u$ of problem (3.1). Furthermore, $T_{k}\left(u_{n}\right)$ converges to $T_{k}(u)$ in $W_{0}^{1, p}(\Omega)$ for any $k>0$. 
We also recall the following estimate [20, Th 2.1].

Theorem 3.4 Let $\Omega$ be a bounded domain of $\mathbb{R}^{N}$. Then there exists a constant $K_{1}>0$, depending on $p$ and $N$ such that if $\mu \in \mathfrak{M}_{b}^{+}(\Omega)$ and $u$ is a nonnegative renormalized solution of problem (3.1) with data $\mu$, there holds

$$
\frac{1}{K_{1}} \mathbf{W}_{1, p}^{\frac{d(x, \partial \Omega)}{3}}[\mu](x) \leq u(x) \leq K_{1} \mathbf{W}_{1, p}^{2 \operatorname{diam}(\Omega)}[\mu](x) \quad \forall x \in \Omega,
$$

where the positive constant $K_{1}$ only depends on $N, p$.

Proof of Theorem 1.1. Let $\left\{u_{m}\right\}_{m \in \mathbb{N}}$ be a sequence of nonnegative renormalized solutions of the following problems

$$
\begin{array}{rlrl}
-\Delta_{p} u_{0} & =\mu & & \text { in } \Omega, \\
u_{0}=0 & & \text { on } \partial \Omega,
\end{array}
$$

and, for $m \in \mathbb{N}$,

$$
\begin{aligned}
-\Delta_{p} u_{m+1} & =P_{l, a, \beta}\left(u_{m}\right)+\mu & & \text { in } \Omega, \\
u_{m+1} & =0 & & \text { on } \partial \Omega .
\end{aligned}
$$

Clearly, we can assume that $\left\{u_{m}\right\}$ is nondecreasing, see [21]. By Theorem 3.4 we have

$$
\begin{aligned}
\chi_{\Omega} u_{0} & \leq K_{1} \mathbf{W}_{1, p}^{R}[\mu], \\
\chi_{\Omega} u_{m+1} & \leq K_{1} \mathbf{W}_{1, p}^{R}\left[P_{l, a, \beta}\left(u_{m}\right)+\mu\right] \quad \forall m \in \mathbb{N},
\end{aligned}
$$

where $R=2 \operatorname{diam}(\Omega)$. Thus, by Theorem 2.5 with $f \equiv 0$, there exists $M>0$ depending on $N, p, l, a, \beta, K_{1}$ and $R$ such that $P_{l, a, \beta}\left(4 c_{p} K_{1} \mathbf{W}_{1, p}^{R}[\omega]\right) \in L^{1}(\Omega)$ and

$$
u_{m}(x) \leq 2 c_{p} K_{1} \mathbf{W}_{1, p}^{R}[\omega](x) \quad \forall x \in \Omega, m \in \mathbb{N},
$$

provided that

$$
\left\|M_{p, R}^{\frac{(p-1)(\beta-1)}{\beta}}[\mu]\right\|_{L^{\infty}\left(\mathbb{R}^{N}\right)} \leq M,
$$

where $\omega=M\left\|\mathbf{M}_{p, R}^{\frac{(p-1)(\beta-1)}{\beta}}[1]\right\|_{L^{\infty}\left(\mathbb{R}^{N}\right)}^{-1}+\mu$ and $c_{p}=1 \vee 4^{\frac{2-p}{p-1}}$. This implies that $\left\{u_{m}\right\}$ is well defined and nondecreasing. Thus $\left\{u_{m}\right\}$ converges a.e in $\Omega$ to some function $u$ which satisfies (1.17) in $\Omega$. Furthermore, we deduce from (3.3) and the monotone convergence theorem that $P_{l, a, \beta}\left(u_{m}\right) \rightarrow P_{l, a, \beta}(u)$ in $L^{1}(\Omega)$. Finally, by Theorem 3.3 we obtain that $u$ is a renormalized solution of (1.16).

Conversely, assume that (1.16) admits a nonnegative renormalized solution $u$. By Theorem 3.4 there holds

$$
u(x) \geq \frac{1}{K_{1}} \mathbf{W}_{1, p}^{\frac{d(x, \partial \Omega)}{3}}\left[P_{l, a, \beta}(u)+\mu\right](x) \text { for all } x \in \Omega .
$$

Hence, we achieve (1.18) from Theorem 2.7, Part 2.

Applications. We consider the case $p=2, \beta=1$. Then $l=2$ and

$$
P_{l, a, \beta}(r)=e^{a r}-1-a r .
$$


If $\Omega$ is a bounded domain in $\mathbb{R}^{N}$, there exists $M>0$ such that if $\mu$ is a positive Radon measure in $\Omega$ which satisfies

$$
\mu\left(B_{t}(x)\right) \leq M t^{N-2} \quad \forall t>0 \text { and almost all } x \in \Omega,
$$

there exists a positive solution $u$ to the following problem

$$
\begin{aligned}
-\Delta u & =e^{a u}-1-a u+\mu & & \text { in } \Omega, \\
u & =0 & & \text { on } \partial \Omega .
\end{aligned}
$$

Furthermore

$$
u(x) \leq K(N) \int_{0}^{2 \operatorname{diam} \Omega} \frac{\omega\left(B_{t}(x)\right)}{t^{N-1}} d t=K(N) \int_{0}^{2 \operatorname{diam}(\Omega)} \frac{\mu\left(B_{t}(x)\right)}{t^{N-1}} d t+b \quad \forall x \in \Omega .
$$

where $b=2 K(N) M \|\left.\mathbf{M}_{2,2 \operatorname{diam}(\Omega)}[1]\right|_{L^{\infty}\left(\mathbb{R}^{N}\right)} ^{-1}\left|B_{1}\right|(\operatorname{diam} \Omega)^{2}$. In the case $N=2$ this result has already been proved by Richard and Véron [22, Prop 2.4].

\section{4 p-superharmonic functions and quasilinear equations in $\mathbb{R}^{N}$}

We recall some definitions and properties of $p$-superharmonic functions.

Definition 4.1 A function $u$ is said to be p-harmonic in $\mathbb{R}^{N}$ if $u \in W_{l o c}^{1, p}\left(\mathbb{R}^{N}\right) \cap C\left(\mathbb{R}^{N}\right)$ and $-\Delta_{p} u=0$ in $\mathcal{D}^{\prime}\left(\mathbb{R}^{N}\right)$. A function $u$ is called a p-supersolution in $\mathbb{R}^{N}$ if $u \in W_{\text {loc }}^{1, p}\left(\mathbb{R}^{N}\right)$ and $-\Delta_{p} u \geq 0$ in $\mathcal{D}^{\prime}\left(\mathbb{R}^{N}\right)$.

Definition 4.2 A lower semicontinuous (l.s.c) function $u: \mathbb{R}^{N} \rightarrow(-\infty, \infty]$ is called p-superharmonic if $u$ is not identically infinite and if, for all open $D \subset \subset \mathbb{R}^{N}$ and all $v \in C(\bar{D})$, p-harmonic in $D, v \leq u$ on $\partial D$ implies $v \leq u$ in $D$.

Let $u$ be a $p$-superharmonic in $\mathbb{R}^{N}$. It is well known that $u \wedge k \in W_{l o c}^{1, p}\left(\mathbb{R}^{N}\right)$ is a p-supersolution for all $k>0$ and $u<\infty$ a.e in $\mathbb{R}^{N}$, thus, $u$ has a gradient (see the previous section). We also have $|\nabla u|^{p-1} \in L_{l o c}^{q}\left(\mathbb{R}^{N}\right), \frac{|\nabla u|^{p}}{(|u|+1)^{r}} \in L_{l o c}^{1}\left(\mathbb{R}^{N}\right)$ and $u \in L_{l o c}^{s}\left(\mathbb{R}^{N}\right)$ for $1 \leq q<\frac{N}{N-1}$ and $r>1$, $1 \leq s<\frac{N(p-1)}{N-p}$ (see [14, Theorem 7.46]). In particular, if $e^{a|u|} \in L_{l o c}^{1}\left(\mathbb{R}^{N}\right)$ for some $a>0$, then $u \in W_{\text {loc }}^{1, p_{1}}\left(\mathbb{R}^{N}\right)$ for all $1 \leq p_{1}<p$ by Hölder's inequality. Thus for any $0 \leq \varphi \in C_{c}^{1}(\Omega)$, by the dominated convergence theorem,

$$
\left\langle-\Delta_{p} u, \varphi\right\rangle=\int_{\mathbb{R}^{N}}|\nabla u|^{p-2} \nabla u \nabla \varphi d x=\lim _{k \rightarrow \infty} \int_{\mathbb{R}^{N}}|\nabla(u \wedge k)|^{p-2} \nabla(u \wedge k) \nabla \varphi \geq 0 .
$$

Hence, by the Riesz Representation Theorem we conclude that there is a nonnegative Radon measure denoted by $\mu[u]$, called Riesz measure, such that $-\Delta_{p} u=\mu[u]$ in $\mathcal{D}^{\prime}\left(\mathbb{R}^{N}\right)$.

The following weak convergence result for Riesz measures proved in 27] will be used to prove the existence of $p$-superharmonic solutions to quasilinear equations. 
Theorem 4.3 Suppose that $\left\{u_{n}\right\}$ is a sequence of nonnegative p-superharmonic functions in $\mathbb{R}^{N}$ that converges a.e to a p-superharmonic function $u$. Then the sequence of measures $\left\{\mu\left[u_{n}\right]\right\}$ converges to $\mu[u]$ in the weak sense of measures.

The next theorem is proved in [20]

Theorem 4.4 Let $\mu$ be a measure in $\mathfrak{M}^{+}\left(\mathbb{R}^{N}\right)$. Suppose that $\mathbf{W}_{1, p}[\mu]<\infty$ a.e. Then there exists a nonnegative p-superharmonic function $u$ in $\mathbb{R}^{N}$ such that $-\Delta_{p} u=\mu$ in $\mathcal{D}^{\prime}\left(\mathbb{R}^{N}\right)$, $\inf _{\mathbb{R}^{N}} u=0$ and

$$
\frac{1}{K_{1}} \mathbf{W}_{1, p}[\mu](x) \leq u(x) \leq K_{1} \mathbf{W}_{1, p}[\mu](x),
$$

for all $x$ in $\mathbb{R}^{N}$, where the constant $K_{1}$ is as in Theorem 3.4. Furthermore any p-superharmonic function $u$ in $\mathbb{R}^{N}$, such that $\inf _{\mathbb{R}^{N}} u=0$ satisfies (4.1) with $\mu=-\Delta_{p} u$.

Proof of Theorem 1.2, Let $\left\{u_{m}\right\}_{m \in \mathbb{N}}$ be a sequence of $p$-superharmonic solutions of the following problems

$$
\begin{aligned}
-\Delta_{p} u_{0} & =\mu \quad \text { in } \quad \mathcal{D}^{\prime}\left(\mathbb{R}^{N}\right), \\
\inf _{\mathbb{R}^{N}} u_{0} & =0,
\end{aligned}
$$

and, for $m \in \mathbb{N}$,

$$
\begin{aligned}
-\Delta_{p} u_{m+1} & =P_{l, a, \beta}\left(u_{m}\right)+\mu \text { in } \mathcal{D}^{\prime}\left(\mathbb{R}^{N}\right), \\
\inf _{\mathbb{R}^{N}} u_{m+1} & =0 .
\end{aligned}
$$

Clearly, we can assume that $\left\{u_{m}\right\}$ is nondecreasing. By Theorem 4.4 we have

$$
\begin{aligned}
u_{0} & \leq K_{1} \mathbf{W}_{1, p}[\mu], \\
u_{m+1} & \leq K_{1} \mathbf{W}_{1, p}\left[P_{l, a, \beta}\left(u_{m}\right)+\mu\right] \quad \forall m \in \mathbb{N} .
\end{aligned}
$$

Thus, by Theorem 2.6 with $f \equiv 0$, there exists $M>0$ depending on $N, p, l, a, \beta, K_{1}$ and $R$ such that $P_{l, a, \beta}\left(4 c_{p} K_{1} \mathbf{W}_{1, p}[\omega]\right) \in L^{1}\left(\mathbb{R}^{N}\right)$ and

$$
u_{m} \leq 2 c_{p} K_{1} \mathbf{W}_{1, p}[\omega] \quad \forall m \in \mathbb{N},
$$

provided that

$$
\left\|M_{p}^{\frac{(p-1)(\beta-1)}{\beta}}[\mu]\right\|_{L^{\infty}\left(\mathbb{R}^{N}\right)} \leq M,
$$

where $\omega=M\left\|\mathbf{M}_{p}^{\frac{(p-1)(\beta-1)}{\beta}}\left[\chi_{B_{R}}\right]\right\|_{L^{\infty}\left(\mathbb{R}^{N}\right)}^{-1} \chi_{B_{R}}+\mu$. This implies that $\left\{u_{m}\right\}$ is well defined and nondecreasing. Thus, $\left\{u_{m}\right\}$ converges a.e in $\mathbb{R}^{N}$ to some $p$-superharmonic function $u$ which satisfies (1.20) in $\mathbb{R}^{N}$. Furthermore, we deduce from (4.2) and the monotone convergence theorem that $P_{l, a, \beta}\left(u_{m}\right) \rightarrow P_{l, a, \beta}(u)$ in $L^{1}\left(\mathbb{R}^{N}\right)$. Finally, by Theorem 4.3 we conclude that $u$ is a p-superharmonic solution of (1.19).

Conversely, assume that (1.19) admits a nonnegative renormalized solution $u$. By Theorem 4.4 there holds

$$
u(x) \geq \frac{1}{K_{1}} \mathbf{W}_{1, p}\left[P_{l, a, \beta}(u)+\mu\right](x) \quad \text { for all } x \in \mathbb{R}^{N} .
$$

Hence, we obtain (1.21) from Theorem 2.7, Part 1, (ii). 


\section{$5 \quad$ Hessian equations}

In this section $\Omega \subset \mathbb{R}^{N}$ is either a bounded domain with a $C^{2}$ boundary or the whole $\mathbb{R}^{N}$. For $k=1, \ldots, N$ and $u \in C^{2}(\Omega)$ the k-hessian operator $F_{k}$ is defined by

$$
F_{k}[u]=S_{k}\left(\lambda\left(D^{2} u\right)\right)
$$

where $\lambda\left(D^{2} u\right)=\lambda=\left(\lambda_{1}, \lambda_{2}, \ldots, \lambda_{N}\right)$ denotes the eigenvalues of the Hessian matrix of second partial derivative $D^{2} u$ and $S_{k}$ is the k-th elementary symmetric polynomial that is

$$
S_{k}(\lambda)=\sum_{1 \leq i_{1}<\ldots<i_{k} \leq N} \lambda_{i_{1}} \ldots \lambda_{i_{k}} .
$$

We can see that

$$
F_{k}[u]=\left[D^{2} u\right]_{k},
$$

where for a matrix $A=\left(a_{i j}\right),[A]_{k}$ denotes the sum of the $\mathrm{k}$-th principal minors. We assume that $\partial \Omega$ is uniformly $(\mathrm{k}-1)$-convex, that is

$$
S_{k-1}(\kappa) \geq c_{0}>0 \text { on } \partial \Omega
$$

for some positive constant $c_{0}$, where $\kappa=\left(\kappa_{1}, \kappa_{2}, \ldots, \kappa_{n-1}\right)$ denote the principal curvatures of $\partial \Omega$ with respect to its inner normal.

Definition 5.1 An upper-semicontinuous function $u: \Omega \rightarrow[-\infty, \infty)$ is $k$-convex ( $k$-subharmonic) if, for every open set $\Omega^{\prime} \subset \bar{\Omega}^{\prime} \subset \Omega$ and for every function $v \in C^{2}\left(\Omega^{\prime}\right) \cap C\left(\overline{\Omega^{\prime}}\right)$ satisfying $F_{k}[v] \leq 0$ in $\Omega^{\prime}$, the following implication is true

$$
u \leq v \text { on } \partial \Omega^{\prime} \Longrightarrow u \leq v \text { in } \Omega^{\prime} .
$$

We denote by $\Phi^{k}(\Omega)$ the class of all k-subharmonic functions in $\Omega$ which are not identically equal to $-\infty$.

The following weak convergence result for $k$-Hessian operators proved in 25] is fundamental in our study.

Theorem 5.2 Let $\Omega$ be either a bounded uniformly $(k-1)$-convex in $\mathbb{R}^{N}$ or the whole $\mathbb{R}^{N}$. For each $u \in \Phi^{k}(\Omega)$, there exist a nonnegative Radon measure $\mu_{k}[u]$ in $\Omega$ such that

$1 \mu_{k}[u]=F_{k}[u]$ for $u \in C^{2}(\Omega)$.

2 If $\left\{u_{n}\right\}$ is a sequence of $k$-convex functions which converges a.e to $u$, then $\mu_{k}\left[u_{n}\right] \rightarrow \mu_{k}[u]$ in the weak sense of measures.

As in the case of quasilinear equations with measure data, precise estimates of solutions of k-Hessian equations with measures data are expressed in terms of Wolff potentials. The next results are proved in [25, 18, 20].

Theorem 5.3 Let $\Omega \subset \mathbb{R}^{N}$ be a bounded $C^{2}$, uniformly (k-1)-convex domain. Let $\varphi$ be a nonnegative continuous function on $\partial \Omega$ and $\mu$ be a nonnegative Radon measure. Suppose that $\mu$ can be decomposed under the form

$$
\mu=\mu_{1}+f
$$


where $\mu_{1}$ is a measure with compact support in $\Omega$ and $f \in L^{q}(\Omega)$ for some $q>\frac{N}{2 k}$ if $k \leq \frac{N}{2}$, or $p=1$ if $k>\frac{N}{2}$. Then there exists a nonnegative function $u$ in $\Omega$ such that $-u \in \Phi^{k}(\Omega)$, continuous near $\partial \Omega$ and $u$ is a solution of the problem

$$
\begin{aligned}
F_{k}[-u] & =\mu & & \text { in } \Omega, \\
u & =\varphi & & \text { on } \partial \Omega .
\end{aligned}
$$

Furthermore, any nonnegative function $u$ such that $-u \in \Phi^{k}(\Omega)$ which is continuous near $\partial \Omega$ and is a solution of above equation, satisfies

$$
\frac{1}{K_{2}} \mathbf{W}_{\frac{2 k}{k+1}, k+1}^{\frac{d(x, \partial \Omega)}{8}}[\mu] \leq u(x) \leq K_{2}\left(\mathbf{W}_{\frac{2 k}{k+1}, k+1}^{2 \operatorname{diam} \Omega}[\mu](x)+\max _{\partial \Omega} \varphi\right),
$$

where $K_{2}$ is a positive constant independent of $x, u$ and $\Omega$.

Theorem 5.4 Let $\mu$ be a measure in $\mathfrak{M}^{+}\left(\mathbb{R}^{N}\right)$ and $2 k<N$. Suppose that $\mathbf{W}_{\frac{2 k}{k+1}, k+1}[\mu]<\infty$ a.e. Then there exists $u,-u \in \Phi^{k}\left(\mathbb{R}^{N}\right)$ such that $\inf _{\mathbb{R}^{N}} u=0$ and $F_{k}[-u]=\mu$ in $\mathbb{R}^{N}$ and

$$
\frac{1}{K_{2}} \mathbf{W}_{\frac{2 k}{k+1}, k+1}[\mu](x) \leq u(x) \leq K_{2} \mathbf{W}_{\frac{2 k}{k+1}, k+1}[\mu](x),
$$

for all $x$ in $\mathbb{R}^{N}$, where the constant $K_{2}$ is the one of the previous Theorem. Furthermore, if $u$ is a nonnegative function such that $\inf _{\mathbb{R}^{N}} u=0$ and $-u \in \Phi^{k}\left(\mathbb{R}^{N}\right)$, then (5.2) holds with $\mu=F_{k}[-u]$.

Proof of Theorem 1.3. We defined a sequence of nonnegative functions $u_{m}$, continuous near $\partial \Omega$ and such that $-u_{m} \in \Phi^{k}(\Omega)$, by the following iterative scheme

$$
\begin{array}{rlrl}
F_{k}\left[-u_{0}\right] & =\mu & & \text { in } \Omega, \\
u_{0}=\varphi & & \text { on } \partial \Omega,
\end{array}
$$

and, for $m \geq 0$,

$$
\begin{aligned}
F_{k}\left[-u_{m+1}\right] & =P_{l, a, \beta}\left(u_{m}\right)+\mu & & \text { in } \Omega, \\
u_{m+1} & =\varphi & & \text { on } \partial \Omega .
\end{aligned}
$$

Clearly, we can assume that $\left\{u_{m}\right\}$ is nondecreasing, see 21. By Theorem 5.3 we have

$$
\begin{aligned}
\chi_{\Omega} u_{0} & \leq K_{2} \mathbf{W}_{\frac{2 k}{k+1}, k+1}^{R}[\mu]+b_{0}, \\
\chi_{\Omega} u_{m+1} & \leq K_{2} \mathbf{W}_{\frac{2 k}{k+1}, k+1}^{R}\left[P_{l, a, \beta}\left(u_{m}\right)+\mu\right]+b_{0},
\end{aligned}
$$

where $b_{0}=K_{2} \max _{\partial \Omega} \varphi$ and $R=2 \operatorname{diam}(\Omega)$.

Then, by Theorem 2.5 with $f=b_{0}$ and $\varepsilon=a$, there exists $M_{1}>0$ depending on $N, k, l, a, \beta, K_{2}$ and $R$ such that $P_{l, a, \beta}\left(4 K_{2} \mathbf{W}_{\frac{2 k}{k+1}, k+1}^{R}\left[\omega_{1}\right]+2 g+b_{0}\right) \in L^{1}(\Omega)$ and

$$
u_{m}(x) \leq 2 K_{2} \mathbf{W}_{\frac{2 k}{k+1}, k+1}^{R}\left[\omega_{1}\right](x)+g+b_{0} \quad \forall x \in \Omega, \forall m \geq 0,
$$

provided that

$$
\left\|M_{2 k, R}^{\frac{k(\beta-1)}{\beta}}[\mu]\right\|_{L^{\infty}\left(\mathbb{R}^{N}\right)} \leq M_{1} \text { and }\left\|M_{2 k, R}^{\frac{k(\beta-1)}{\beta}}\left[P_{l, 2 a, \beta}\left(b_{0}\right)\right]\right\|_{L^{\infty}\left(\mathbb{R}^{N}\right)} \leq M_{1},
$$


where $\omega_{1}=M_{1}\left\|\mathbf{M}_{2 k}^{\frac{(p-1)(\beta-1)}{\beta}}[1]\right\|_{L^{\infty}\left(\mathbb{R}^{N}\right)}^{-1}+\mu, \omega_{2}=M_{1}\left\|\mathbf{M}_{2 k}^{\frac{(p-1)(\beta-1)}{\beta}}[1]\right\|_{L^{\infty}\left(\mathbb{R}^{N}\right)}^{-1}+P_{l, 2 a, \beta}\left(b_{0}\right)$ and $g=2 K_{2} \mathbf{W}_{\frac{2 k}{k+1}, k+1}^{R}\left[\omega_{2}\right]$.

Since $\omega_{2}$ is constant, $g$ has the same property and actually $g=K_{2}\left(\left|B_{1}\right| \omega_{2}\right)^{\frac{1}{k}} R^{2}$. On the other hand, one can find constants $M_{2}$ depending on $N, k, l, a, \beta, R$ and $M_{1}$ such that if $\max _{\partial \Omega} \varphi \leq M_{2}$, then $\left\|M_{2 k, R}^{\frac{k(\beta-1)}{\beta}}\left[P_{l, 2 a, \beta}\left(b_{0}\right)\right]\right\|_{L^{\infty}\left(\mathbb{R}^{N}\right)} \leq M_{1}$.

Hence, we deduce from (5.6) that $P_{l, a, \beta}\left(2 K_{2} \mathbf{W}_{\frac{2 k}{k+1}, k+1}^{R}[\mu]+b\right) \in L^{1}(\Omega)$ and

$$
u_{m}(x) \leq 2 K_{2} \mathbf{W}_{\frac{2 k}{k+1}, k+1}^{R}[\mu](x)+b \quad \forall x \in \Omega, \forall m \geq 0,
$$

for some constant $b\left(=2 g+b_{0}\right)$ depending on $N, k, l, a, \beta, R$ and $M_{1}$. Note that because we can write

$$
\omega=P_{l, a, \beta}\left(u_{m}\right)+\mu=\left(\mu_{1}+\chi_{\Omega_{\delta}} P_{l, a, \beta}\left(u_{m}\right)\right)+\left(\left(1-\chi_{\Omega_{\delta}}\right) P_{l, a, \beta}\left(u_{m}\right)+f\right),
$$

where $\Omega_{\delta}=\{x \in \Omega: d(x, \partial \Omega)>\delta\}$ and $\delta>0$ is small enough and since $u_{m}$ is continuous near $\partial \Omega$, then $\omega$ satisfies the assumptions of the data in Theorem [5.3. Therefore the sequence $\left\{u_{m}\right\}$ is well defined and nondecreasing. Thus, $\left\{u_{m}\right\}$ converges a.e in $\Omega$ to some function $u$ for which (1.23) is satisfied in $\Omega$. Furthermore, we deduce from (5.7) and the monotone convergence theorem that $P_{l, a, \beta}\left(u_{m}\right) \rightarrow P_{l, a, \beta}(u)$ in $L^{1}(\Omega)$. Finally, by Theorem 5.2, we obtain that $u$ satisfies (1.22) and (1.23).

Conversely, assume that (1.22) admits nonnegative solution $u$, continuous near $\partial \Omega$, such that $-u \in \Phi^{k}(\Omega)$ and $P_{l, a, \beta}(u) \in L^{1}(\Omega)$. Then by Theorem 5.3 we have

$$
u(x) \geq \frac{1}{K_{2}} \mathbf{W}_{\frac{2 k}{k+1}, k+1}^{\frac{d(x, \partial \Omega)}{8}}\left[P_{l, a, \beta}(u)+\mu\right](x) \quad \text { for all } x \in \Omega .
$$

Using the part 2 of Theorem 2.7 we conclude that (1.24) holds.

Proof of Theorem 1.4. We define a sequence of nonnegative functions $u_{m}$ with $-u_{m} \in$ $\Phi^{k}\left(\mathbb{R}^{N}\right)$, by the following iterative scheme

$$
\begin{aligned}
& F_{k}\left[-u_{0}\right]=\mu \\
& \inf _{\mathbb{R}^{N}} u_{0}=0,
\end{aligned}
$$

and, for $m \geq 0$,

$$
\begin{aligned}
& F_{k}\left[-u_{m+1}\right]=P_{l, a, \beta}\left(u_{m}\right)+\mu \quad \text { in } \mathbb{R}^{N} \\
& \inf _{\mathbb{R}^{N}} u_{m+1}=0 .
\end{aligned}
$$

Clearly, we can assume that $\left\{u_{m}\right\}$ is nondecreasing. By Theorem 5.4, we have

$$
\begin{aligned}
u_{0} & \leq K_{2} \mathbf{W}_{\frac{2 k}{k+1}, k+1}[\mu], \\
u_{m+1} & \leq K_{2} \mathbf{W}_{\frac{2 k}{k+1}, k+1}\left[P_{l, a, \beta}\left(u_{m}\right)+\mu\right] .
\end{aligned}
$$

Thus, by Theorem 2.6 with $f \equiv 0$, there exists $M>0$ depending on $N, k, l, a, \beta$ and $R$ such that $P_{l, a, \beta}\left(4 K_{2} \mathbf{W}_{\frac{2 k}{k+1}, k+1}[\omega]\right) \in L^{1}\left(\mathbb{R}^{N}\right)$,

$$
u_{m} \leq 2 K_{2} \mathbf{W}_{\frac{2 k}{k+1}, k+1}[\omega] \quad \forall m \geq 0,
$$


provided that $\left\|M_{2 k}^{\frac{k(\beta-1)}{\beta}}[\mu]\right\|_{L^{\infty}\left(\mathbb{R}^{N}\right)} \leq M$, where $\omega=M\left\|\mathbf{M}_{2 k}^{\frac{k(\beta-1)}{\beta}}\left[\chi_{B_{R}}\right]\right\|_{L^{\infty}\left(\mathbb{R}^{N}\right)}^{-1} \chi_{B_{R}}+\mu$.

Therefore the sequence $\left\{u_{m}\right\}$ is well defined and nondecreasing. By arguing as in the proof of theorem 1.3 we obtain that $u$ satisfies (1.25) and (1.26).

Conversely, assume that (1.25) admits a nonnegative solution $u$ and $-u \in \Phi^{k}\left(\mathbb{R}^{N}\right)$ such that $P_{l, a, \beta}(u) \in L_{l o c}^{1}\left(\mathbb{R}^{N}\right)$, then by Theorem 5.4 we have

$$
u \geq \frac{1}{K_{2}} \mathbf{W}_{\frac{2 k}{k+1}, k+1}\left[P_{l, a, \beta}(u)+\mu\right] .
$$

Using the part 1, (ii) of Theorem 2.7, we conclude that (1.27) holds.

\section{References}

[1] D. R. Adams, L.I. Heberg, Function Spaces and Potential Theory, Grundlehren der Mathematischen Wisenschaften 31, Springer-Verlag (1999).

[2] D.R. Adams, M. Pierre, Capacitary strong type estimates in semilinear problems, Ann. Inst. Fourier (Grenoble) 41 (1991), 117-135.

[3] P. Baras, M. Pierre, Critère d'existence des solutions positives pour des équations semilinéaires non monotones, Ann. Inst. H. Poincaré, Anal. Non Lin. 3 (1985), 185-212.

[4] M. F. Bidaut-Véron, Local and global behavior of solutions of quasilinear equations of Emden-Fowler type, Arch. Ration. Mech. Anal. 107 (1989), 293-324.

[5] M. F. Bidaut-Véron, Necessary conditions of existence for an elliptic equation with source term and measure data involving p-Laplacian, in: Proc. 2001 Luminy Conf. on Quasilinear Elliptic and Parabolic Equations and Systems, Electron. J. Differ. Equ. Conf. 8 (2002), 23-34.

[6] M. F. Bidaut-Véron, Removable singularities and existence for a quasilinear equation with absorption or source term and measure data, Adv. Nonlinear Stud. 3 (2003), 25-63.

[7] M. F. Bidaut-Véron, S. Pohozaev, Nonexistence results and estimates for some nonlinear elliptic problems, J. Anal. Math. 84 (2001), 1-49.

[8] M. F. Bidaut-Véron, H. Nguyen Quoc, L. Véron, Quasilinear Lane-Emden equations with absorption and measure data, to appear in Journal des Mathématiques Pures et Appliquées (2014).

[9] I. Birindelli, F. Demengel, Some Liouville theorems for the p-Laplacian, in: Proc. 2001 Luminy Conf. on Quasilinear Elliptic and Parabolic Equations and Systems, Electron. J. Differ. Equ. Conf. 8 (2002), 35-46.

[10] G. Dal Maso, F. Murat, L. Orsina, A. Prignet, Renormalized solutions of elliptic equations with general measure data, Ann. Sc. Norm. Sup. Pisa, 28 (1999), 741-808.

[11] R. Fefferman, Strong differentiation with respect to measure, Amer. J. Math 103 (1981), 33-40.

[12] F. Ferrari, B. Franchi, I. Verbitsky, Hessian inequality and the fractional Laplacian, J. Reine Angew. Math. 667 (2012), 133-148. 
[13] N. Grenon. Existence results for semilinear elliptic equations with small measure data., Ann. Inst. H. Poincaré, Anal. Non Lin. 19 (2002), 1-11.

[14] J. Heinonen, T. Kilpelinen, O. Martio, Nonlinear potential theory of degenerate elliptic equations. Unabridged republication of the 1993 original. Dover Publications, Inc., Mineola, NY, 2006. xii+404 pp.

[15] T. Kilpelainen, J. Malý, Degenerate elliptic equation with measure data and nonlinear potentials, Ann. Sc. Norm. Super. Pisa, Cl. Sci. 19 (1992), 591-613.

[16] T. Kilpelainen, J. Malý, The Wiener test and potential estimates for quasilinear elliptic equations, Acta Math. 172 (1994), 137-161.

[17] P. Honzik, B. Jaye, On the good-入 inequality for nonlinear potentials, Proc. Amer. Math. Soc. 140, 4167-4180, (2012).

[18] D. Labutin, Potential estimates for a class of fully nonlinear elliptic equations, Duke Math. J. 111, 1-49, (2002).

[19] M. M. Rao, Z. D. Ren, Theory of Orlicz Spaces, Textbooks in Pure and Applied Mathematics (1991).

[20] N. C. Phuc, I. E. Verbitsky, Quasilinear and Hessian equations of Lane-Emden type, Ann. Math. 168, 859-914 (2008).

[21] N. C. Phuc, I. E. Verbitsky, Singular quasilinear and Hessian equation and inequalities, J. Functional Analysis 256, 1875-1906 (2009).

[22] Y. Richard, L. Véron, Isotropic singularities of solutions of nonlinear elliptic inequalities, Ann. I.H.P. Analyse Non Linéaire 6, 37-72 (1989).

[23] J. Serrin, H. Zou, Cauchy-Liouville and universal boundedness theorems for quasilinear elliptic equations and inequalities, Acta Math. 189(2002) 79-142.

[24] N. S. Trudinger and X.J. Wang, Hessian measures, Topological Methods in Nonlinear Analysis 10 (1997), 225-239.

[25] N. S. Trudinger, X.J. Wang, Hessian measures II, Annals of Mathematics 150 (1999), 579-604.

[26] N. S. Trudinger, X. J. Wang, Hessian measures III, Journal of Functional Analysis 193 (2002), 1-23.

[27] N. S. Trudinger, X. J. Wang, On the weak continuity of elliptic operators and applications to potential theory, Amer. J. Math. 124 (2002), 369-410.

[28] L. Véron, Elliptic equations involving measures, in Stationary Partial Differential Equations, vol. I, Handbook of Equations, Elsevier B.V., pp. 593-712 (2004). 